\title{
AS CONDIÇÕES DE SAÚDE AFETAM OS RENDIMENTOS DO TRABALHO? EVIDÊNCIAS PARA O MERCADO DE TRABALHO NO BRASIL
}

\author{
Wallace Patrick Santos de Farias Souza * \\ Flávio Augusto ZiegelmanN ${ }^{\dagger}$ \\ Erik Alencar de Figueiredo
}

\begin{abstract}
Resumo
O estudo busca mensurar o efeito das condições de saúde sobre o rendimento dos trabalhadores brasileiros, usando dados da PNAD de 2008. Para tanto, foram estimados o efeito de tratamento médio e quantílico com correção para o viés de seleção; o método de Lewbel (2012) que gera instrumentos internos a partir da heterocedasticidade dos resíduos e bounds para o efeito do tratamento seguindo Oster (2015). Os resultados mostram um impacto negativo de uma má condição de saúde na renda do trabalho para todas as especificações utilizadas, sendo o maior impacto sofrido por indivíduos localizados nos quantis mais baixos de renda.
\end{abstract}

Palavras-chave: condição de saúde; efeito de tratamento; renda do trabalho

\begin{abstract}
The study aims to measure the effect of health conditions on the Brazilian workers income, using PNAD data from 2008. For that, we estimated: the average and quantile treatment effect with correction for the selection bias; the method of Lewbel (2012), that generates internal instruments from heteroscedasticity of residues; and bounds for the treatment effect following Oster (2015). The results show a negative impact of a poor health condition on labor income for all the specifications used, with the greatest impact being suffered by individuals located in the lowest income quantiles.
\end{abstract}

Keywords: health condition; treatment effect; labor income JEL classification: $\mathrm{C} 21, \mathrm{I} 10, \mathrm{~J} 22$

DOI: http://dx.doi.org/10.11606/1980-5330/ea132569

\footnotetext{
* Programa de Pós Graduação em Economia - UFPB. E-mail:wpsfarias@gmail.com

† Programa de Pós Graduação em Economia - UFRGS. E-mail: flavioz@ufrgs.br

‡ Programa de Pós Graduação em Economia - UFPB. E-mail: eafigueiredo@gmail.com
} 


\section{Introdução}

O acesso à saúde é considerado um dos principais fatores de acumulação de capital humano e influencia positivamente na função de bem-estar dos indivíduos, dado que quanto maior seu estoque de saúde maior a disponibilidade para o trabalho, estudo e/ou lazer. A condição saudável é também considerada um fim em si mesmo, pois só o fato de ter uma boa saúde contribui para o aumento do bem-estar dos indivíduos. As condições de saúde afetam os rendimentos dos trabalhadores na medida em que influenciam a sua decisão em participar ou não da força de trabalho, o número de horas trabalhadas e a sua produtividade. Dessa forma, indivíduos saudáveis possuem um maior estoque de capital humano e tendem a ser mais produtivos quando comparados aos seus pares não saudáveis. (Alves et al. 2002).

A saúde, diferente de outras formas de capital humano, tem uma importância peculiar para o retorno econômico, pois as condições físicas e mentais que o indivíduo se encontra determinam a quantidade e intensidade com que os mesmos podem se dedicar a atividade laboral, contribuindo para o aumento dos rendimentos e bem-estar (Grossman 1972). Nesse sentido, trabalhos pioneiros como Schultz (1961) e Mincer (1974) apontam saúde e educação como componentes principais do capital humano.

Fatores não controláveis, como herança genética, e controláveis, como hábitos saudáveis, definem o estoque de saúde dos indivíduos, e este determina o quanto de trabalho os indivíduos estão dispostos e são capazes de ofertar. $\mathrm{O}$ tipo de atividade é também importante para determinar a quantidade de trabalho ofertada, pois a presença de qualquer doença que gere limitações físicas poderá ter um impacto maior em tarefas que exijam um maior esforço físico, que por sua vez, são normalmente tarefas desempenhadas com menor grau de qualificação (Noronha et al. 2004).

Grossman (1972) introduz a ideia de que a procura de saúde é resultado de um processo de escolha individual. Assim, os cuidados médicos são entendidos como um fator produtivo adquirido pelos indivíduos para produzir saúde. Para o autor, a saúde é um estoque, tendo duração de vários anos, e tal estoque se depreciaria com o passar do tempo. O modelo proposto pelo autor evidencia que a procura de cuidados médicos terá um objetivo último que é a procura de saúde e, além disso, a procura por cuidados médicos é influenciada por fatores sócioeconômicos que afetam o estado de saúde, tais como, as preferências individuais, o salário, a idade, o nível educacional.

A grande maioria dos trabalhos que analisam os determinantes do retorno salarial do indivíduo focam na educação como componente de capital humano, tal como na formulação de Mincer (1974). Outros trabalhos como Bartel \& Taubman (1979), Levine et al. (1997), Kassouf (1997), Alves \& Andrade (2003) e Böckerman et al. (2015), acrescentam um componente de saúde na formulação minceriana, sobre a hipótese que o estoque de saúde afeta a produtividade do trabalho e oferta de mão de obra.

Para o Brasil, apesar de ser uma área recente de pesquisa, alguns trabalhos se destacam como referência na análise do impacto da saúde no resultado econômico individual. Kassouf (1999), por exemplo, verifica que um IMC ${ }^{1}$ baixo (usado como indicador de subnutrição) tem um impacto significativo sobre o rendimento dos indivíduos para o Brasil, enquanto que Alves \& An-

\footnotetext{
${ }^{1}$ Índice de Massa Corporal.
} 
drade (2003), em estudo para o Estado de Minas Gerais, avaliam que as más condições de saúde tem como efeitos a redução salarial ou até a saída do indivíduo do mercado de trabalho.

A maioria dos artigos identificam três canais pelos quais as condições de saúde afetam as decisões dos indivíduos no mercado de trabalho e consequentemente os ganhos auferidos por tais trabalhadores: a participação na força de trabalho, o número de horas trabalhadas e o nível de salário. Com respeito à oferta de trabalho, a saúde tem impactos sobre a escolha setorial, sobre a decisão de trabalhar e sobre número de horas a ser ofertado (Strauss \& Thomas 1998). No entanto, o resultado de uma má condição de saúde sobre a oferta de trabalho pode ser ambíguo, dependendo de três efeitos, a saber: efeito dotação ${ }^{2}$, efeito substituição ${ }^{3}$ e efeito renda ${ }^{4}$. Se, por exemplo, o efeito renda superar o efeito dotação mais o efeito substituição juntos, uma saúde precária resulta em maior oferta de trabalho. Mas no geral, é esperado que aconteça o contrário, e que um trabalhador doente tende a trabalhar menos tempo do que uma pessoa saudável ${ }^{5}$.

Por outro lado, indivíduos que possuem doenças (sobretudo crônicas) buscam se adaptar ao ambiente de trabalho e executar normalmente suas tarefas, a não ser que a doença seja grave o suficiente que o impeça de trabalhar. Para saber se de fato o estado de saúde implica em restrições capazes de reduzir a produtividade e perdas salariais, deve ser levada em conta a gravidade da enfermidade e a capacidade de adaptação do indivíduo.

Com relação ao número de horas trabalhadas por semana, esta é dada pela soma das horas semanais alocadas para o trabalho principal, secundário e outros. Porém, esse número de horas assim como o nível de rendimento é observado apenas para aqueles que participam da força de trabalho, de maneira que a amostra utilizada na estimação das equações não é aleatória, dado que indivíduos que não participam da força de trabalho não aparecem na amostra selecionada. Dessa forma, é preciso um método que corrige a seletividade amostral, observando quais variáveis afetam a probabilidade de um determinado indivíduo entrar no mercado de trabalho. Muitos trabalhos aplicados tem usado a correção do viés de seleção baseado no procedimento de Heckman (1979), segundo o qual as motivações que os indivíduos têm para ocupar postos de trabalho podem diferir entre os indivíduos, levando em conta suas características.

Em suma, as condições de saúde podem afetar a participação no mercado de trabalho, o nível de salário por hora individual via efeitos sobre a produtividade e a quantidade de horas trabalhadas pelos indivíduos. Para o presente trabalho, o foco será no efeito que as condições de saúde exercem sobre os rendimentos do trabalho, sendo a variável dependente o salário por hora semanal auferido, em logaritmo.

Dito isso, o objetivo deste artigo é mensurar o impacto das condições de

\footnotetext{
${ }^{2}$ Refere-se a menor quantidade de tempo para realizar as atividades, inclusive trabalhar, quando está doente.

${ }^{3}$ Quando a condição de saúde precária corresponde a uma taxa de salário menor, fazendo com que o indivíduo substitua trabalho por lazer (reduza sua oferta de trabalho), dado que o trabalho gera desutilidade.

${ }^{4}$ Quando a condição de saúde precária faz o indivíduo trabalhar mais para compensar a menor taxa de salário por hora.

${ }^{5} \mathrm{~A}$ intensidade com que essa redução acontece pode depender, entre outros fatores, do tipo de doença.
} 
saúde dos indivíduos no seu nível salarial, a partir dos microdados da Pesquisa Nacional por Amostra de Domicílios para o ano de 2008, que contem um suplemento de saúde dos entrevistados ${ }^{6}$. Para alcançar tal objetivo, as estimativas serão baseadas em quatro estratégias empíricas, como forma de tentar mitigar a possibilidade de variáveis não observadas estarem viesando os resultados. A primeira delas é estimar o efeito de tratamento médio das condições de saúde sobre a renda do trabalho por meio do propensity score matching, como forma de ter estimativas mais razoáveis que as obtidas por $\mathrm{OLS}^{7}$, tendo como tratamento ${ }^{8}$ uma má condição de saúde autorreportada ou ser diagnosticado com alguma doença crônica em comparação a indivíduos saudáveis (grupo de controle). No entanto, é possível que a condição de saúde tenha um efeito diferente ao longo da distribuição de salários e o efeito médio não represente completamente o seu impacto, visto que uma condição de saúde insatisfatória pode ter um reflexo maior para aquelas ocupações que necessitam de maior esforço físico, que em geral são exercidas por indivíduos pouco qualificados e, consequentemente, com baixa remuneração. Assim, o segundo método empírico estimado é o efeito por quantis, como forma de ver o impacto do tratamento (status de saúde) por toda a distribuição de salários. Para os dois casos é feita uma estimação em primeiro estágio para corrigir o viés de seleção, verificando as variáveis que afetam a probabilidade do indivíduo estar inserido no mercado de trabalho.

Além do efeito heterogêneo entre os quantis, um baixo nível de salário implica que os indivíduos têm menos recursos para cuidar da sua condição de saúde (exames, diagnósticos, prevenções, etc) tendo como consequência um nível baixo desse indicador. Em outras palavras, a condição de saúde pode afetar no rendimento do trabalho assim como o nível de renda pode afetar na condição de saúde (causalidade reversa). Além disso, a relação ainda pode apresentar a endogeneidade em decorrência de viés de variável relevante omitida ou via erro de medida, fazendo com que a condição de saúde não seja exógena. Nesse caso, uma alternativa seria a necessidade de uma variável instrumental que afete o status de saúde dos indivíduos (tratamento), mas não impacte diretamente na renda do trabalho (resultado) para contornar esse problema. Dado a ausência de instrumentos externos disponíveis (ou ser muito difícil justificar e/ou encontrar bons instrumentos), a terceira estratégia usada é um estimador de dois estágios proposto por Lewbel (2012) que explora a heterocedasticidade do primeiro estágio para gerar instrumentos internos para identificação. Por fim, na quarta estratégia serão estimados os bounds para o efeito de tratamento desenvolvido por Oster (2015) que informa sobre o viés que persiste devido a variáveis não observadas, dada à preocupação com variáveis omitidas na estimação do coeficiente de interesse.

O artigo apresenta a seguinte estrutura, além desta introdução. A seção seguinte traz um resumo das evidências empíricas acerca da influência das condições de saúde no mercado de trabalho. Na Seção 3 é feita uma análise do estado de saúde no Brasil, segundo a amostra utilizada, a descrição da base de dados e a criação das variáveis de saúde usadas como tratamento. A quarta seção apresenta todos os passos da estratégia empírica adotada, bem como

\footnotetext{
${ }^{6}$ Os detalhes e a justificativa sobre a utilização da base de dados utilizada será explicitado na Seção 3.

${ }^{7}$ Ordinary least squares.

${ }^{8} \mathrm{~A}$ construção das variáveis de tratamento estão explicadas na seção de dados.
} 
o método de correção do viés de seleção. Em seguida são apresentados os resultados encontrados e, por fim, na sexta seção são feitas as considerações finais.

\section{Revisão de Literatura}

Vários trabalhos empíricos têm sido realizados para tentar entender a relação das condições de saúde e mercado de trabalho, seja usando a saúde como uma variável exógena tal qual em Luft (1975) e Kassouf (1999), ou tentando controlar a endogeneidade entre saúde e rendimentos tal como Grossman \& Benham (1974) e Schultz \& Tansel (1997). O trabalho de Luft (1975) mensura as perdas de salários ocasionadas por uma má condição de saúde dos trabalhadores americanos entre 18 e 64 anos, diferenciando por sexo e raça. Kassouf (1999) utiliza o mesmo procedimento para o Brasil mensurando as condições de saúde por meio do estado nutricional individual, avaliando também as diferenças regionais e entre as áreas urbana e rural.

Segundo Gomes et al. (2014), normalmente indivíduos com menores rendimentos desempenham atividades que exigem maior esforço físico que intelectual, o que fundamenta a relação dos rendimentos condicionados ao estado de saúde. Dessa forma, os autores identificam um "círculo vicioso" em tal relação, bo qual os indivíduos mais pobres tendem a ocupar trabalhos que requerem maior esforço e consequentemente maior perda de rendimentos quando se encontram doentes (devido à impossibilidade de empreender o esforço), o que podem torná-los mais pobres e agravar mais ainda a condição de saúde. Assim, a presença de qualquer tipo de doença que gere limitações físicas poderá ter um impacto maior sobre os rendimentos dos indivíduos que desempenham tarefas que demandem mais esforço físico, quando comparadas a atividades que requeiram um menor nível de esforço Noronha et al. (2004). Em outras palavras, visto que a remuneração pode ser definida pela produtividade, indivíduos com condições de saúde precária tendem a ser menos produtivos. Isso implica que irão auferir uma menor remuneração, a depender do tipo de doença.

Nesse sentido, usando equações simultâneas para dados da Austrália da AusDiab Survey (ADS), Cai (2010) verificou que os dois efeitos podem ser encontrados. A saúde afeta a participação da força de trabalho e, por outro lado, à participação da força de trabalho afeta a saúde, sendo tal efeito negativo para os homens e positivo para as mulheres.

Smith (1999) também discute sobre a direção da causalidade entre medidas de renda e variáveis de saúde, visto que para os países industrializados o argumento de que um menor status socioeconômico leva a um menor cuidado de saúde ou a um maior padrão de comportamento prejudicial à saúde, parece insuficiente para justificar essa relação. Com isso, têm surgido teorias que tentam explicar o canal pelo qual o baixo nível socioeconômico pode levar a piora das condições de saúde, tais como os impactos de longo prazo na primeira infância, fatores ambientais intrauterinos ou a exposição prolongada a fatores estressantes. Por outro lado, uma má condição de saúde pode reduzir a capacidade dos indivíduos de obter renda, impossibilitar para o trabalho ou aumentar as despesas médicas, impactando na renda disponível dos mesmos. $\mathrm{O}$ autor ainda enfatiza que a saúde pode alterar a poupança das famílias (e consequentemente a riqueza), estimando a magnitude desses efeitos. 
Dessa forma, o trabalho de Smith adverte sobre a superestimação de causalidade em uma direção, dado que pode haver uma simultaneidade. Suas conclusões mostram que a renda parece afetar o status de saúde de forma mais incisiva na infância e no início da idade adulta, quando o estoque e nível de saúde ainda está sendo estabelecido. Já em idades mais avançadas, há um efeito mais pronunciado da condição de saúde na renda e riqueza.

Barnay (2016) faz uma revisão da literatura empírica para países europeus acerca da relação entre emprego e estado de saúde, mostrando que são encontrados efeitos nos dois sentidos, tanto da saúde nos resultados do trabalho quanto do emprego no estado de saúde. Controlando por questões de endogeneidade (causalidade reversa, variáveis omitidas) a literatura tem mostrado que um ambiente de trabalho favorável e segurança no emprego levam a uma melhor condição de saúde. Por outro lado, o desemprego e a aposentadoria causam, em geral, uma piora na saúde mental dos trabalhadores. Dentre as observações apontadas na pesquisa, é mostrado que cerca de $29 \%$ dos trabalhadores europeus gostariam de ter uma jornada de trabalho menor na tentativa de reduzir efeitos prejudiciais sobre a saúde. No geral, uma combinação de elevada proteção do emprego, satisfação profisisonal e políticas de mercado de trabalho implicam em um efeito positivo nas condições de saúde dos trabalhadores. O autor chama ainda a atenção para o fato de que os trabalhadores europeus enfrentaram uma degradação da qualidade de vida entre 1995 e 2005, quando quase um terço dos cidadãos na União Europeia apontaram que o trabalho afeta sua saúde. Isso fez com que ao longo do tempo demandassem uma maior rede de proteção social contra choques inesperados de saúde.

Em âmbito internacional, alguns trabalhos tem usado painel de dados quando a disponibilidade dos mesmos torna possível, tais como Disney et al. (2006) e García-Gómez et al. (2010). Disney et al. (2006) estimam um painel de dados da British Household Panel Survey (BHPS) de 1991-1998 para o Reino Unido e encontra que choques negativos nas condições de saúde tem impacto sobre a aposentadoria precoce dos trabalhadores e consequentemente um menor tempo no mercado de trabalho, enquanto que García-Gómez et al. (2010) usando dados da mesma pesquisa para 1991-2002 mostram que a saúde afeta a entrada e saída do emprego.

Por outro lado, Morris (2007) aplica vários métodos (propensity score mathcing, probit, probit bivariado com variável instrumental) para dados da Health Survey for England de 1997-1998 e verifica um impacto negativo da obesidade sobre o nível de emprego, enfocando que a estimativa que não corrige a endogeneidade com o uso do instrumento é submestimada para as mulheres. Harris (2009) também encontra efeitos negativos da diabetes e doenças cardiovasculares na participação no mercado de trabalho para a Austrália em 2002.

É oportuno frisar que a relação entre saúde e renda do trabalho pode persistir no tempo, dado que o status de saúde tende a se deteriorar com a idade. Por outro lado, os indivíduos podem se adaptar a nova condição ou buscar atividades mais adaptáveis ${ }^{9}$ ao seu estado de saúde. Contudo, esses efeitos ainda não são consolidados na literatura. Dito isso, García-Gómez et al. (2013) analisam os efeitos de longo prazo das condições de saúde no emprego e renda para

\footnotetext{
${ }^{9} \mathrm{~A}$ adaptação do indivíduo a trabalhos que requerem habilidades compatíveis com sua capacidade residual após o choque negativo de saúde depende das características individuais e do sistema de seguro de invalidez. García-Gómez et al. (2013) mostram que esse sistema de seguro nos Países Baixos estavam entre os mais generosos do mundo.
} 
uma amostra de internações hospitalares e dados de imposto de renda para a Holanda (amostra de indivíduos entre 18 e 64 anos de idade para um período de 8 anos) e verificam, a partir de métodos de propensity score matching e diferenças em diferenças, que os efeitos negativos ainda persistem de 2 até 6 anos após um indivíduo ter sido internado com uma doença grave. Além do efeito negativo na probabilidade de emprego e na renda do indivíduo afetado (internado), há um efeito de transbordamento maior na renda familiar. Como a internação não é prevista (mais provável ser exógena que uma medida de saúde autorreportada) e a amostra contém apenas indivíduos não internados no ano anterior, os autores evitam o problema da causalidade reversa e, aliado a estratégia empírica adotada, fornecem resultados mais robustos.

García-Gómez et al. (2013) também estimam o efeito da internação do indivíduo sobre a oferta de trabalho do cônjuge e encontram que, em média, a probabilidade de o cônjuge estar trabalhando é reduzida e a renda é menor mesmo dois anos após a admissão hospitalar. Tal efeito é heterogêneo entre os gêneros, sendo que quando o homem fica doente é mais provável que a mulher continue ou entre no mercado de trabalho ao passo que, quando a mulher tem um choque negativo de saúde, existe uma probabilidade maior de o homem deixar a força de trabalho. O efeito é maior ainda entre os idosos quando comparado a outras faixas de idade, dada a menor capacidade de recuperação após o choque.

Usando registros de dados de trabalhadores suecos, Lundborg et al. (2015) também encontram evidências de heterogeneidade do impacto da saúde sobre os rendimentos do trabalho para indivíduos com alto e baixo nível de educação. Os indivíduos podem ser acompanhados por até 12 anos, podendo considerar o impacto heterogêneo do choque de saúde tanto de curto e longo prazos. Além disso, os dados sobre os choques inesperados trazem estimativas com menos viés que as notificações de condições de saúde autorreportadas. A partir de métodos de diferença em diferença, os resultados mostram que um mesmo choque de saúde não esperado tem um efeito negativo maior para trabalhadores com menor nível de escolaridade e que esse efeito aumenta com a idade. Uma das explicações é que indivíduos mais educados têm maior consciência sobre cuidados com a saúde, e mesmo após um choque têm maior facilidade em aderir a tratamentos médicos. Do ponto de vista social, é de interesse que indivíduos mais qualificados retornem mais rapidamente ao mercado de trabalho dados os custos sociais e de seguridade. Os autores ainda revelam que os trabalhadores pouco qualificados também são mais propensos a deixar a força de trabalho, receber seguro invalidez e auxílios doença após o choque negativo nas condições de saúde. Impactos na fertilidade e na renda do cônjuge são também analisados.

Os resultados heterogêneos encontrados por Lundborg et al. (2015) são uma explicação sobre o gradiente educacional em saúde, que representa as diferenças de saúde entre os diferentes grupos da sociedade. Portanto, os autores mostram que esse padrão é encontrado na Suécia, um país com cobertura universal de seguro de saúde e cuidados médicos de alta qualidade. Outros trabalhos como Case \& Deaton (2005) e Van Kippersluis et al. (2009) têm evidenciado a presença do gradiente durante a idade ativa dos indivíduos, diminuindo à medida que as pessoas envelhecem.

Para o Brasil, Alves \& Andrade (2003) encontram efeitos diferenciados da saúde nos rendimentos de homens e mulheres no mercado de trabalho para Minas Gerais. Para os homens, o principal efeito de uma má condição de 
saúde é a exclusão do mercado de trabalho, enquanto que para as mulheres é a redução na taxa de salários.

Seguindo essa linha, Godoy et al. (2006) encontram efeitos negativos na renda individual decorrente de doença renal crônica a partir dos dados da PNAD de 1998, impactando com mais intensidade trabalhadores de baixa renda. A hipótese dos autores é que além de impactos no mercado de trabalho propriamente dito ${ }^{10}$, a doença renal tem outras implicações econômicas como aposentadoria precoce, aumento de programas assistenciais de transferência de renda e custos elevados do sistema de saúde. Cuidados com prevenção e o retardamento dessa e outras doenças estão entre as recomendações para a redução das disparidades de rendimentos no mercado de trabalho. Silva et al. (2011) encontra resultados semelhantes com relação à tuberculose, que implica em perdas salarias para os trabalhadores, sobretudo para os mais pobres.

Outros trabalhos para grupos específicos também podem ser encontrados na literatura nacional. Pérez et al. (2006), por exemplo, analisam os determinantes da participação dos idosos no mercado de trabalho para o ano $2000 \mathrm{em}$ São Paulo. Para controlar o viés de endogeneidade entre saúde e participação no mercado de trabalho, foram utilizadas variáveis que buscaram captar as condições e acesso a serviços de saúde durante a infância dos indivíduos e outras para controlar o comportamento nocivo a saúde como instrumentos. Os resultados mostram que uma pior condição de saúde reduz a probabilidade de trabalhar, sendo esse efeito maior para os homens. Isso se deve ao fato de as mulheres estarem inseridas em atividades que demandem um menor esforço físico, fazendo com que a condição de saúde não seja tão crucial. Das demais variáveis utilizadas, o nível socioeconômico tem uma maior importância para explicar a oferta de trabalho dos homens, enquanto para as mulheres são os atributos ligados à composição familiar que têm maior relevância. Uma possível explicação, segundo os autores apud Saad (1999), é que as mulheres mantêm laços familiares mais fortes.

Quando considerados no agregado, os efeitos das condições de saúde sobre os rendimentos individuais podem influenciar as condições macroeconômicas. Posto isso, Figueirêdo et al. (2003) verificaram durante a década de 1990 que um bom estado de saúde da população contribui positivamente para o crescimento econômico, seja por meio da taxa de crescimento do PIB ou reduções na taxa de mortalidade. Os autores argumentam que mais investimentos em capital humano podem ampliar tais efeitos.

Em suma, a literatura tem corroborado que uma má condição de saúde afeta negativamente a participação no mercado de trabalho, seja pela exclusão do trabalhador ou redução do rendimento e jornada de trabalho.

\section{Base de Dados}

\subsection{Análise do Estado de Saúde no Brasil}

A Pesquisa Nacional por amostra de Domicílios de 2008 traz um suplemento com informações sobre o estado de saúde dos indivíduos, que pode ser mensurado a partir de vários aspectos e critérios. Seguindo a literatura e a disponibilidade de dados, a avaliação empírica do estado de saúde pode ser au-

${ }^{10}$ Tais como redução de salários e de número de horas trabalhadas. 
topercebida, baseada em conhecimentos e crenças pessoais, ou reportada por um profissional de saúde, observando a incidência de uma série de doenças crônicas que os indivíduos possam apresentar. São também utilizadas variáveis que indicam se o indivíduo apresenta problemas de realizar tarefas ou se locomover e ainda aspectos comportamentais relacionados à saúde, como tabagismo e sedentarismo/atividade física.

Com relação à autoavaliação reportada pelo indivíduo, este é um critério subjetivo que, no entanto, capta a percepção do indivíduo sobre sua real disposição em realizar determinadas tarefas, além de ser um indicador sobre o estado de saúde global. Uma desvantagem é que isso pode estar relacionado com características socioeconômicas e culturais das pessoas.

Já os indicadores de dificuldades de mobilidade ou limitações funcionais, apesar de também serem autoreportados, estão menos sujeitos à subjetividade, pois focam em perguntas específicas sobre determinadas tarefas corriqueiras, que o indivíduo tem consciência sobre o seu real desempenho. Um problema é que tais dificuldades apresentam baixa frequência, sobretudo na população mais jovem, o que torna difícil captar o estado de saúde nessa faixa etária.

A Tabela 1 apresenta o estado de saúde autoavaliado, no qual o indivíduo é questionado como em geral classifica seu próprio estado de saúde, sendo possíveis cinco respostas (muito bom, bom, regular, ruim, muito ruim). É percebido que enquanto $3 \%$ dos entrevistados classificam seu estado de saúde ruim ou muito ruim, mais de $70 \%$ o classificam como muito bom ou bom, chegando a quase $80 \%$ para os homens ${ }^{11}$.

O alto percentual de pessoas que consideram sua condição de saúde como boa (muito boa) pode indicar o desconhecimento em relação a alguma doença que não foi diagnosticada ou à própria relutância em admitir alguma enfermidade, sobretudo para os homens, menos propensos a realizar consultas de rotina e cuidados gerais com a sua saúde.

Tabela 1: Autoavaliação do estado de saúde individual PNAD (2008) - em \%

\begin{tabular}{lccr}
\hline Autoavaliação de Saúde & Homens & Mulheres & Total \\
\hline Muito Bom & 23,07 & 19,66 & 21,35 \\
Bom & 54,85 & 53,79 & 54,32 \\
Regular & 19,51 & 23,33 & 21,44 \\
Ruim & 2,23 & 2,60 & 2,42 \\
Muito Ruim & 0,34 & 0,62 & 0,48 \\
\hline
\end{tabular}

Fonte: Elaboração dos autores com base nos dados da PNAD (2008).

Outro critério a ser utilizado é o clínico/funcional avaliado por um profissional de saúde, um indicador importante para avaliar o estado de saúde individual pelo fato de fornecer uma informação objetiva para mensurar a saúde. No entanto, se o indivíduo não tem costume de ir ao médico ou fazer exames periódicos, pode ser que desconheça a existência de algum problema

\footnotetext{
${ }^{11}$ Para fins metodológicos, como será detalhado mais adiante, tal variável será transformada em binária, que assume valor 0 para os indivíduos que apresentam o estado de saúde autodeclarado como "muito bom" ou "bom", e 1 para indivíduos que apresentam o estado "regular", "ruim" ou "muito ruim".
} 
de saúde que possa ter, principalmente aqueles que não apresentam sintomas claros em fases iniciais.

Entre as doenças avaliadas, são observadas as de características como alta prevalência (hipertensão), altas taxas de utilização de serviços de saúde (bronquite e asma), possibilidade de intervenção efetiva (tuberculose) e elevada frequência de uso de serviços de alto custo (insuficiência renal crônica, câncer). Ao todo são 12 doenças para as quais os indivíduos são ou não diagnosticados em tê-las por um profissional de saúde, sendo o percentual de indivíduos identificados com cada uma dessas doenças reportado na Tabela 2. É importante salientar que os indivíduos podem apresentar mais de uma doença, fato não destacado na tabela, mas que será captado pela criação de uma variável referente ao número de doenças ${ }^{12}$.

Pela Tabela 2, é possível perceber que doenças relacionadas à coluna e hipertensão são as mais frequentes na população brasileira, enquanto que cirrose e tuberculose afetam uma parcela muito pequena. Quanto à diferença de gênero, as mulheres representam a maioria do total de afetados para praticamente todas as doenças, chegando a $76 \%$ do total de pessoas com depressão. Apenas a tuberculose e a cirrose afetam uma proporção maior de homens, esta última chegando a mais de $71 \%$ do total de entrevistados com a doença.

Tabela 2: Indivíduos diagnosticados com alguma doença - PNAD (2008) em \%

\begin{tabular}{lccr}
\hline Doenças & $\begin{array}{c}\text { Homens } \\
\text { (\% Total) }\end{array}$ & $\begin{array}{r}\text { Mulheres } \\
\text { (\% Total) }\end{array}$ & Total \\
\hline Coluna & 43,62 & 56,38 & 19,27 \\
Artrite & 33,61 & 66,39 & 6,22 \\
Câncer & 37,10 & 62,90 & 0,46 \\
Diabetes & 48,42 & 51,58 & 3,26 \\
Hipertensão & 43,42 & 56,58 & 15,76 \\
Coração & 44,22 & 55,78 & 3,44 \\
Insuficiência Renal & 44,23 & 55,77 & 1,52 \\
Depressão & 23,41 & 76,59 & 5,27 \\
Tuberculose & 53,57 & 46,43 & 0,14 \\
Tendinite & 24,11 & 75,89 & 4,61 \\
Cirrose & 71,23 & 28,77 & 0,12 \\
\hline
\end{tabular}

Fonte: Elaboração dos autores com base nos dados da PNAD (2008).

Uma mesma doença pode afetar as pessoas com diferentes intensidades e causar diferentes percepções quanto ao estado de saúde individual. Dessa forma, a Tabela 3 indica como os indivíduos diagnosticados com alguma doença classificam seu estado de saúde. Para todas as doenças, observa-se que a maioria dos indivíduos diagnosticados reporta seu estado de saúde como bom ou regular o que pode indicar a perda de rendimentos ou redução do número de horas trabalhadas, mas não a exclusão do mercado de trabalho. Esse fato é corroborado pela estatística descritiva mais adiante, em que a quase totalidade de indivíduos da amostra está inserida no mercado de trabalho.

\footnotetext{
${ }^{12}$ A criação dessa variável será explicada mais adiante.
} 
Tabela 3: Autoavaliação do estado de saúde de indivíduos diagnosticados com alguma doença - PNAD (2008) em \%

\begin{tabular}{lcccrc}
\hline Doenças & Muito Bom & Bom & Regular & Ruim & Muito Ruim \\
\hline Coluna & 9,57 & 42,38 & 40,18 & 6,49 & 1,38 \\
Artrite & 5,63 & 33,67 & 47,71 & 10,65 & 2,34 \\
Câncer & 9,54 & 34,28 & 43,11 & 12,01 & 1,06 \\
Diabetes & 4,77 & 31,76 & 51,08 & 9,48 & 2,91 \\
Hipertensão & 6,64 & 41,93 & 43,17 & 6,75 & 1,52 \\
Coração & 4,76 & 27,10 & 51,93 & 12,93 & 3,28 \\
Insuficiência Renal & 5,72 & 31,07 & 49,08 & 11,22 & 2,91 \\
Depressão & 7,06 & 34,82 & 44,64 & 10,91 & 2,58 \\
Tuberculose & 13,10 & 42,86 & 35,71 & 8,33 & 0,00 \\
Tendinite & 13,85 & 43,47 & 34,94 & 6,14 & 1,60 \\
Cirrose & 9,59 & 38,36 & 31,51 & 17,81 & 2,74 \\
\hline
\end{tabular}

Fonte: Elaboração dos autores com base nos dados da PNAD (2008).

As dificuldades de mobilidade e/ou locomoção, a criação das variáveis binárias que serão usadas como tratamento e as demais características individuais e de localidade usadas como controle serão descritas junto com as principais estatísticas descritivas abaixo.

\subsection{Base de Dados}

A base de dados utilizada neste estudo são os microdados da Pesquisa Nacional por Amostra de Domicílios (PNAD 2008) realizada pelo Instituto Brasileiro de Geografia e Estatística (IBGE) para o ano de 2008. Além das informações permanentes sobre características socioeconômicas domiciliares e pessoais (como renda, idade, raça, sexo, renda per capita, etc.), esse ano investiga características do levantamento suplementar de saúde. O suplemento é uma fonte de dados importante para o conhecimento e monitoramento de aspectos relevantes da situação de saúde da população brasileira e do acesso, utilização e financiamento de serviços de saúde, assim como informações sobre mobilidade, sedentarismo, entre outros.

A utilização dessa base de dados se deu pelo fato de a PNAD englobar uma amostra representativa do território nacional, ser maior em comparação a outras pesquisas de saúde, o questionário ser respondido para todas as pessoas do domicílio, bem como a disponibilidade de outras variáveis de controle, como é o caso de rendas das mais diversas fontes (aposentadorias, pensões, lucros), dado que não é uma pesquisa puramente de saúde. Além disso, outros trabalhos da literatura nacional também fizeram uso do suplemento de saúde da PNAD de 2008, servindo como robustez para a estratégia empírica adotada no presente estudo. É sabido que outras pesquisas sobre saúde podem ser encontradas, como a Pesquisa Nacional de Saúde (PNS) para o ano de 2013, que inclusive se utiliza da amostra mestra da PNAD contínua. No entanto, continuaria sendo uma "fotografia" retirada em um ponto no tempo, o que achamos não ter muitas mudanças significativas. É importante ainda frisar que mais que o valor do coeficiente, a estratégia buscou apresentar indícios do efeito causal de uma má condição de saúde na perda de rendimento do trabalho dos indivíduos. Uma vez encontradas evidências de tal efeito, é razoável supor que este independa de um período de tempo específico, a não ser 
que se considere períodos demasiadamente longos com mudanças estruturais no mercado de trabalho e indicadores da economia.

Foram utilizados dois critérios para classificar os indivíduos entre doentes ou saudáveis, além de variáveis usadas como controle que indicam a existência de dificuldade de problemas de locomoção e execução de tarefas domésticas e corriqueiras. Tais critérios foram recodificados para variáveis binárias e usados como tratamento para diferenciar os indivíduos quanto ao impacto na renda do trabalho e à participação no mercado de trabalho.

O primeiro critério é a condição de saúde autoavaliada, que, embora seja uma avaliação subjetiva dos próprios indivíduos, dá uma noção de percepção global do estado de saúde e é consistente com diferentes medidas autorreportadas. As respostas variam de 1 a 5 de acordo com a seguinte classificação: $1=$ "muito boa", 2= "boa", 3= "regular", 4= "ruim", 5= "muito ruim". Dessa forma, ao contrário de Noronha et al. (2004), foi criada uma variável dummy que assume valor um se indivíduo relata sua condição de saúde como "regular", "ruim" ou "muito ruim", e assume valor zero para uma condição "boa" ou "muito boa", fazendo com que o grupo de controle seja saudável ${ }^{13}$.

O outro critério de saúde adotado como tratamento é a presença de doença diagnosticada por um profissional de saúde, sendo uma variável dummy que assume valor um se o indivíduo foi diagnosticado com uma ou mais doenças e zero caso não tenha nenhuma doença. Como robustez, foram construídas dummies (também usadas como tratamento em outras estimativas, uma por vez) para verificar se uma pessoa possui mais de uma doença, ou seja, uma variável binária assumindo valor um se possui duas ou mais doenças e zero caso saudável, e por fim, uma variável binária que assume valor um se o indivíduo possui três ou mais doenças e zero caso não tenha sido diagnosticado com nenhuma doença.

O critério subjetivo como um indicador da percepção global que o indivíduo tem a respeito de seu estado de saúde, possivelmente, mensura de forma mais adequada o estado de saúde do indivíduo do que o critério clínico/funcional, e estaria captando de forma mais ampla as restrições que o estado de saúde impõe ao desempenho dos doentes no trabalho, sobretudo, sobre sua produtividade.

Com relação a restrição de atividades rotineiras por motivo de saúde referese a comportamentos geralmente associados à redução de atividades que a pessoa é capaz de desempenhar no seu dia a dia. Tais restrições ${ }^{14}$ foram transformadas em variáveis binárias que assumem o valor um se o indivíduo apresenta dificuldade para aquela característica e zero caso contrário, sendo depois agrupadas em uma variável que representa o número de problemas/dificuldades de realizar atividades e/ou locomoção que o indivíduo enfrenta. Tal variável foi usada como controle em todas as estimações.

Características pessoais (sexo, raça, idade, escolaridade, hábitos saudáveis) relacionadas à região (UF, área metropolitana, etc) e informações sobre o setor e ramo de atividade no mercado de trabalho foram também usadas como con-

\footnotetext{
${ }^{13}$ A definição como foi exposto é para padronizar com a outra variável binária de condição de saúde, que assume valor 1 se o indivíduo é diagnosticado com alguma doença, fazendo o grupo de controle novamente ser saudável.

${ }^{14}$ As características de locomoção são relacionadas ao fato de o indivíduo ter: dificuldade em alimentar-se, dificuldade de levantar objetos, dificuldade em realizar consertos domésticos, dificuldade em subir ladeira ou escada, dificuldade em abaixar-se, dificuldade em andar mais de 100 metros, dificuldade para andar mais de um quilômetro.
} 
troles. Por fim, para a equação de participação no mercado de trabalho que corrige o viés de seleção, além das características pessoais e de região citadas, foram utilizadas variáveis de renda do não trabalho (pensão, juros, aposentadorias, aluguel, etc), que são incluídas por afetarem o salário de reserva dos agentes e a decisão de entrar ou não no mercado de trabalho ${ }^{15}$, ou seja, os indivíduos exigem salários de reserva maiores para trocar lazer por trabalho. Estas são as variáveis de exclusão do modelo, dado que afetam a probabilidade de um indivíduo ofertar ou não trabalho, mas não afetam diretamente os rendimentos do trabalho ${ }^{16}$.

Além das variáveis relacionadas às condições de saúde já apresentadas, a Tabela 4 apresenta as principais estatísticas descritivas da amostra utilizada, em um total de 60.812 observações sobre indivíduos da PNAD de 2008. É observado que a quase totalidade dos indivíduos está inserida de alguma forma no mercado de trabalho, sendo esta a variável dependente da equação de participação ( $1^{\circ}$ Estágio) que corrige o viés de seleção amostral. Para a equação de interesse ( $2^{\circ}$ Estágio), a renda do trabalho usada para construir o logaritmo do salário por hora apresenta uma média de $\mathrm{R} \$ 1.211,93$. Na Tabela 4, são também marcadas as variáveis usadas em cada um dos estágios.

Os diferentes tipos de renda do não trabalho apresentam uma média muito baixa e um grande desvio padrão, devido ao fato de muitos indivíduos não receberem tais tipos de rendimentos. Com relação aos atributos pessoais, há um maior percentual de pessoas não brancas e uma leve participação a mais de mulheres na amostra, com uma média de idade e escolaridade de 40 anos e 9,23 anos de estudo, respectivamente. Foram excluídas pessoas com menos de 15 e mais de 65 anos de idade, com o intuito de captar apenas os indivíduos com idade ativa para o trabalho, o que faz a média de idade e escolaridade ser um pouco elevada em comparação com a amostra completa. Ainda é notado que do total da amostra, cerca de $28 \%$ praticam alguma atividade física e $20 \%$ são fumantes, sendo hábitos que podem influenciar no status de saúde.

Dentre os aspectos relacionados ao mercado de trabalho, a maioria encontrase no comércio e na indústria de transformação, totalizando $30 \%$ dos trabalhadores. Com relação ao status da posição de ocupação, os trabalhadores com carteira assinada e por conta própria possuem uma representação em torno de $31 \%$ e $27 \%$, respectivamente. Por fim, como é de se esperar, a grande maioria dos indivíduos reside na área urbana $(86 \%)$ e nas regiões Sudeste e Nordeste do país.

\section{Estratégia Empírica}

Esta seção apresenta a estratégia empírica adotada no estudo. Em primeiro lugar será descrito o método de efeito de tratamento médio por propensity score matching, bem como suas limitações e o tratamento por quantis, além da correção do viés de seleção para o primeiro estágio. Em seguida, na Subseção 4.2, será apresentado o método de Lewbel (2012), que usa instrumentos internos a partir da heterocedasticidade, quando não existem restrições de exclusão disponíveis para gerar as estimativas. Por fim, a Subseção 4.3 apresenta o método

\footnotetext{
${ }^{15}$ Quanto maior a renda do não trabalho, maior tenderá a ser o salário de reserva das pessoas, que consequentemente esperam um salário maior para aceitar uma ocupação.

${ }^{16}$ Ao contrário do método de correção proposto por Heckman (1979), será utilizado um método semiparamétrico em que tal restrição não é obrigatoriamente necessária. Mesmo assim, foram utilizadas as rendas do não trabalho, dada a justificativa teórica.
} 
Tabela 4: Estatísticas descritivas - PNAD - 2008

\begin{tabular}{|c|c|c|c|c|c|}
\hline Variáveis & Média & Desvio-padrão & Mínimo & Máximo & Estágio \\
\hline $\begin{array}{l}\text { Renda do Trabalho } \\
\text { (Dependente) }\end{array}$ & $1.211,93$ & $1.772,65$ & 0 & 27.992 & $2^{\circ}$ \\
\hline Trabalho & 0,9714 & 0,1666 & 0 & 1 & $1^{\circ}$ \\
\hline \multicolumn{6}{|c|}{ Renda do Não Trabalho } \\
\hline Pensão & 145,992 & $1.522,472$ & 0 & 10.000 & $1^{\mathrm{o}}$ \\
\hline Aposentadoria & 0,6679 & 418,254 & 0 & 5.700 & $1^{\mathrm{o}}$ \\
\hline Aluguel & 135,666 & $1.682,745$ & 0 & 12.000 & $1^{\mathrm{o}}$ \\
\hline Doação & 23,748 & 574,578 & 0 & 6.000 & $1^{\circ}$ \\
\hline Juros & 110,819 & 760,204 & 0 & 5.000 & $1^{\circ}$ \\
\hline \multicolumn{6}{|c|}{ Características Pessoais } \\
\hline Sexo & 0,5039 & 0,4999 & 0 & 1 & $1^{\circ}, 2^{\circ}$ \\
\hline Raça & 0,4564 & 0,4981 & 0 & 1 & $1^{\circ}, 2^{\circ}$ \\
\hline Idade & 40,6810 & 10,1470 & 25 & 64 & $1^{\circ}, 2^{\circ}$ \\
\hline Estado Civil & 0,7334 & 0,4421 & 0 & 1 & $1^{\circ}, 2^{\circ}$ \\
\hline Anos de Estudo & 9,2320 & 4,5570 & 1 & 16 & $1^{\circ}, 2^{\circ}$ \\
\hline \multicolumn{6}{|c|}{ Hábitos de Saúde } \\
\hline Atividade Física & 0,2885 & 0,4530 & 0 & 1 & $1^{\circ}, 2^{\circ}$ \\
\hline Fumante & 0,2009 & 0,4007 & 0 & 1 & $1^{\circ}, 2^{\circ}$ \\
\hline \multicolumn{6}{|c|}{ Ramo de Atividade } \\
\hline Agrícola & 0,1093 & 0,3120 & 0 & 1 & $2^{\circ}$ \\
\hline Transformação & 0,1374 & 0,3443 & 0 & 1 & $2^{\circ}$ \\
\hline Construção & 0,0624 & 0,2419 & 0 & 1 & $2^{\circ}$ \\
\hline Outras Indústrias & 0,0075 & 0,0865 & 0 & 1 & $2^{\circ}$ \\
\hline Comércio & 0,1627 & 0,3691 & 0 & 1 & $2^{\circ}$ \\
\hline Serviços & 0,0439 & 0,2084 & 0 & 1 & $2^{\circ}$ \\
\hline Serviços Auxiliares & 0,0529 & 0,2239 & 0 & 1 & $2^{\circ}$ \\
\hline Trans. e Comunicação & 0,0434 & 0,2039 & 0 & 1 & $2^{\circ}$ \\
\hline Social & 0,1300 & 0,3363 & 0 & 1 & $2^{\circ}$ \\
\hline Adm. Pública & 0,0697 & 0,2546 & 0 & 1 & $2^{\circ}$ \\
\hline Outras & 0,0807 & 0,2724 & 0 & 1 & $2^{\circ}$ \\
\hline \multicolumn{6}{|c|}{ Posição na Ocupação do Trabalho } \\
\hline Militar & 0,1110 & 0,3142 & 0 & 1 & $2^{\circ}$ \\
\hline Com Carteira & 0,3127 & 0,4636 & 0 & 1 & $2^{\circ}$ \\
\hline Sem Carteira & 0,1472 & 0,3543 & 0 & 1 & $2^{\circ}$ \\
\hline Doméstico & 0,0995 & 0,2994 & 0 & 1 & $2^{\circ}$ \\
\hline Conta-própria & 0,2751 & 0,4465 & 0 & 1 & $2^{\circ}$ \\
\hline Empregador & 0,0542 & 0,2266 & 0 & 1 & $2^{\circ}$ \\
\hline
\end{tabular}

Fonte: Elaboração própria com base nos dados da PNAD 2008. Nota: categorias das variáveis - sexo $(1=$ feminimo; $0=$ masculino $)$, raça $(1=$ brancos; $0=$ brancos $)$. 
Tabela 4: Estatísticas descritivas - PNAD - 2008 (continuação)

\begin{tabular}{lccccc}
\hline Variáveis & Média & Desvio-padrão & Mínimo & Máximo & Estágio \\
\hline $\begin{array}{l}\text { Renda do Trabalho } \\
\text { (Dependente) }\end{array}$ & $1.211,93$ & $1.772,65$ & 0 & 27.992 & $2^{\circ}$ \\
Trabalho & 0,9714 & 0,1666 & 0 & 1 & $1^{\circ}$ \\
\hline \multicolumn{5}{c}{ Localização } \\
\hline Urbana & 0,8634 & 0,3433 & 0 & 1 & $1^{\circ}, 2^{\circ}$ \\
Norte & 0,1298 & 0,3361 & 0 & 1 & $1^{\circ}, 2^{\circ}$ \\
Nordeste & 0,2854 & 0,4516 & 0 & 1 & $1^{\circ}, 2^{\circ}$ \\
Sul & 0,1664 & 0,3724 & 0 & 1 & $1^{\circ}, 2^{\circ}$ \\
Sudeste & 0,2980 & 0,4574 & 0 & 1 & $1^{\circ}, 2^{\circ}$ \\
Centro-Oeste & 0,1201 & 0,3251 & 0 & 1 & $1^{\circ}, 2^{\circ}$ \\
\hline
\end{tabular}

Fonte: Elaboração própria com base nos dados da PNAD 2008. Nota: categorias das variáveis - sexo $(1=$ feminimo; $0=$ masculino $)$, raça $(1=$ brancos; $0=$ brancos $)$.

de (Oster 2013, 2015) para estimar bounds para o efeito de tratamento, como robustez para as estimações anteriores.

\subsection{Efeito de Tratamento}

O interesse do presente trabalho é estimar o efeito causal das condições de saúde sobre as perdas de rendimento do trabalho no Brasil, para indivíduos que reportam seu estado de saúde como ruim ou que têm alguma doença diagnosticada por um profissional de saúde (efeito nos tratados), usando dados do suplemento de saúde da PNAD, como destacado anteriormente. Para tanto, como especificação tradicional para estimar tal efeito, tem-se

$$
Y=\alpha+\beta S+X \gamma+\varepsilon
$$

em que $Y$ é o log da renda do trabalho (salário) por hora, $S$ é um indicador (dummy) para saúde, $X$ é um vetor de variáveis de controle que afetam os salários, e $\varepsilon$ é um termo de erro.

Como mencionado na seção de dados, serão utilizados vários indicadores de saúde como variável binária de tratamento $(S)$, um por vez para cada estimação, a saber: i) dummy = 1 para má condição de saúde autoavaliada e 0 caso contrário; ii) dummy $=1$ para indivíduo diagnosticado com pelo menos uma doença por um profissional de saúde e 0 caso contrário; iii) dummy = 1 para o indivíduo diagnosticado com duas ou mais doenças e 0 com nenhuma doença; iv) $d u m m y=1$ para indivíduo diagnosticado com três ou mais doenças e 0 com nenhuma doença; e v) dummies para cada uma das doze doenças listadas nos dados, sendo 1 = tem a doença e $0=$ não tem a doença. Assim, controlando para um vetor de características observáveis, a estratégia desenvolvida busca observar se existe um efeito de o indivíduo ter uma má condição de saúde $(S)$ no seu nível de salário $(Y)$.

Mensurar $\beta$ de forma consistente requer que o termo de erro não esteja correlacionado com a variável de saúde $(\operatorname{COV}(S ; \varepsilon)=0)$, ou seja, que os indivíduos sejam distribuídos aleatoriamente pelas condições de saúde (condicionado às variáveis observadas), e não satisfazer tal condição torna as estimativas por OLS viesadas e inconsistentes (Angrist \& Pischke 2009). Quando a 
hipótese não se mantém e a seleção do tratamento é baseada em variáveis não observadas correlacionadas com a variável de interesse $(S)$, uma das soluções seria encontrar uma variável instrumental para o efeito causal da condição de saúde que não seja correlacionada com o resultado. No entanto, como nem sempre encontrar um instrumento é trivial, é necessária uma estratégia de identificação que consiga estimar o efeito de interesse da condição de saúde sobre o rendimento do trabalho.

Em outras palavras, busca-se comparar dois grupos de indivíduos com características semelhantes de modo que a única diferença entre eles seja a condição de saúde, sendo que os indivíduos de um grupo foram diagnosticados com alguma doença ou reportaram seu estado de saúde como insatisfatório (dependendo de qual variável está sendo usada na estimação) e o outro grupo com indivíduos saudáveis quanto a esses critérios. No entanto, é possível que a condição de saúde dos indivíduos possa ser afetada por características não observáveis, que mesmo quando controlar pelo vetor $X$ não conseguem ser captadas. Dessa forma, a estratégia empírica aqui presente tenta lidar com essa e outras questões para tentar captar a do rendimento que de fato é afetada pelo status de saúde dos trabalhadores.

A primeira estratégia adotada, comumente usada para balancear variáveis em busca de resultados mais precisos, é o estimador por propensity score matching. Dado o conjunto de variáveis disponíveis, estimar o escore de propensão associado à condição de saúde reduz a influência de variáveis potenciais omitidas.

Seguindo Rosenbaum \& Rubin (1983), denota-se $Y_{i}$ como o resultado observado do indivíduo $i$ para a variável de resultado, o log do salário por hora, e $Y_{1}$ e $Y_{0}$ são os resultados potenciais para o grupo de tratamento (má condição de saúde ou diagnosticado com alguma doença) e que não possui a característica do tratamento (grupo de controle), respectivamente. Dessa forma, tem-se

$$
Y_{i}=S_{i} Y_{i}^{1}+\left(1-S_{i}\right) Y_{i}^{0} .
$$

De acordo com Imbens \& Wooldridge (2009), sabe-se que não se podem comparar os dois resultados para um mesmo indivíduo em um mesmo período de tempo, sendo observado apenas um dos dois resultados potenciais dado o status do tratamento, $Y_{i}=Y_{i}^{0}+\left(Y_{i}^{1}-Y_{i}^{0}\right) S_{i}$. Então, é preciso encontrar indivíduos pertencentes aos dois grupos que possam ser comparados (tratados e controle), depois de ajustadas às características observadas contidas em $X_{i}$ (Angrist \& Pischke 2009).

Tal comparação é justamente a intuição dos estimadores de correspondência, em que, condicionando as variáveis $X_{i}$, os resultados potenciais são independentes do tratamento ${ }^{17}$. Ou seja, $Y_{i}^{1}, Y_{i}^{0} \perp S_{i} \mid X_{i}$, que implica em $Y_{i}^{1}, Y_{i}^{0} \perp S_{i} \mid p\left(X_{i}\right)$, em que $p\left(X_{i}\right)$ é a probabilidade de um indivíduo ser tratado ou o propensity score. Isso permite encontrar o efeito de tratamento para cada valor de $X_{i}$ como a diferença das médias dos dois status de resultado, usando para isso o propensity score para obter o efeito sobre os indivíduos tratados (Rosenbaum \& Rubin 1985). Assim, o ATT ${ }^{18}$ pode ser obtido por

$$
\beta_{A T T}=E\left(Y_{i}^{1}-Y_{i}^{0}\right)
$$

\footnotetext{
${ }^{17}$ Hipótese de independência condicional. Para mais detalhes ver (Rubin 1974, Heckman \& Robb Jr 1985).

${ }^{18}$ Do inglês Average Treatment Effect on the Treated.
} 


$$
\beta_{A T T}=E\left[E\left[Y_{i} \mid p\left(X_{i}\right), S_{i}=1\right]-E\left[Y_{i} \mid p\left(X_{i}\right), S_{i}=0\right]\right]
$$

Para obter o $\beta$ na Equação 4, estima-se o escore de propensão utilizando um modelo probit, fazendo o matching do grupo de tratamento com o grupo de controle a partir do Kernel Matching. Todas as estimações serão feitas em dois estágios, sendo que o primeiro estágio é usado para correção do viés de seleção, observando variáveis que alteram a probabilidade do indivíduo estar ou não inserido no mercado de trabalho ${ }^{19}$.

O kernel Matching usado no pareamento constrói a distribuição contrafactual do resultado para indivíduos com uma má condição de saúde (grupo de tratamento) utilizando uma média local das observações não tratadas (indivíduos saudáveis), cujos escores de propensão estão dentro do intervalo das estimativas de escore de propensão para observações do grupo de tratamento. Os pesos positivos associados às observações de comparação com escores de propensão similares diminuem quanto maior a distância do escore de propensão, garantindo que os maiores pesos sejam atribuídos às observações mais semelhantes. Foi também utilizado o nearest neighbor matching estimator, que para o grupo de tratamento, seleciona um (ou mais, em caso de empate) indivíduo saudável com o escore de propensão mais próximo daquele indivíduo inicial. O pareamento é feito com reposição e empates que recebem o mesmo peso, como é comum na literatura. Os resultados para os diferentes métodos de pareamento não apresentaram diferenças significativas, de modo que nos resultados foram apresentados apenas uma estimativa.

No entanto, o efeito médio pode não refletir completamente a influência do tratamento sobre os resultados potenciais, visto que a distribuição da variável dependente pode ser diferente na parte superior da PORÇÃO inferior da distribuição, dependendo das variáveis utilizadas (o que faz sentido quando se trata de rendimentos). Em outras palavras, é razoável imaginar que indivíduos com um menor nível salarial possam sofrer um impacto diferente daqueles que têm um alto salário, visto que os primeiros terão menos recursos disponíveis para cuidados com sua saúde. Além disso, trabalhos com menor nível de remuneração requerem, em geral, maior esforço físico. Dada uma condição de saúde insatisfatória, supõe-se que estes serão mais afetados.

Dito isso, será estimado também o efeito do tratamento quantílico (QTEs) ${ }^{20}$, dado seu poder intuitivo e a vantagem em permitir caracterizar o efeito em toda a distribuição condicional da variável resposta a partir de um conjunto de regressores. Além dessa vantagem, de acordo com Koenker (n.d.) apud Silva et al. (2011) a utilização da regressão quantílica também pode ser usada quando a distribuição não é gaussiana; a regressão é robusta a outliers por utilizar a distribuição condicional da variável resposta; os estimadores podem ser mais eficientes que os obtidos por meio de OLS, dado que os erros não possuem uma distribuição normal; entre outras. É importante salientar ainda, que as mesmas variáveis de controle, de resultado e tratamento serão utilizadas na estimação do efeito por quantis. O estimador do efeito quantílico do tratamento pode ser usado com regressores endógenos, quando tiver instrumentos disponíveis, ou pode usar o status do tratamento exógeno, sendo reduzida ao estimador de regressão quantílica padrão.

\footnotetext{
${ }^{19}$ A Subseção 4.1 apresenta o método usado para corrigir o viés de seleção, tanto no caso do tratamento médio como no efeito de tratamento quantílico.

${ }^{20}$ Do inglês quantile treatment effects.
} 
Para a descrição do estimador, tem-se que os quantis condicionais dos resultados potenciais para os compliers ${ }^{21}$ são dados por $Q_{\tau}=\left(Y_{i}^{0} \mid X_{i}, S_{i}\right)=X^{\prime} \beta_{\tau} \mathrm{e}$ $Q_{\tau}=\left(Y_{i}^{1} \mid X_{i}, S_{i}\right)=\alpha_{\tau}+X^{\prime} \beta_{\tau}$, em que $\tau$ refere-se ao $\tau$-ésimo quantil pertencente ao intervalo $(0,1)$. Assim sendo, dada a variação exógena do tratamento nos resultados potenciais, a função quantílica condicional pode ser escrita como

$$
Q_{\tau}=\left(Y \mid X_{i}, S_{i}, S_{1}>S_{0}\right)=\alpha_{\tau} S+X^{\prime} \beta_{\tau}
$$

$\mathrm{O} \alpha_{\tau}$ pode representar uma relação causal que mostra a diferença entre o $\tau$-ésimo quantil dos resultados potenciais $\left(Y_{i}^{1}\right.$ e $\left.Y_{i}^{0}\right)$ para os grupos de tratamento e controle, respectivamente. Em outras palavras, o coeficiente $\alpha_{\tau}$ indica se houve mudança no rendimento do trabalho em decorrência da condição de saúde individual.

Como no efeito médio, o tratamento também pode ser correlacionado com o termo de erro o que produziria estimativas viesadas. Em uma primeira estimativa, assume-se que tanto o tratamento $S$, quanto $X$ são exógenos, ou seja, $\varepsilon \perp(S, X)^{22}$. Dessa forma, tem-se que $Q_{y \mid X, S}^{\tau}=X^{\prime} \beta_{\tau}+\alpha_{\tau} S$, tal que é possível recuperar os parâmetros desconhecidos dos resultados potenciais da distribuição conjunta de $y, X$ e $S$, e a estimação pode ser feita pela regressão quantílica clássica como sugerida por Koenker \& Bassett Jr (1978), tal como

$$
\left(\hat{\beta}^{\tau}, \hat{\delta}^{\tau}\right)=\operatorname{argmin}_{\beta, \delta} \sum W_{i} \times \rho_{\tau}\left(y_{i}-X_{i} \beta-H_{i} \delta\right)
$$

em que $\rho_{\tau}=u \times\{\tau-1(u<0)\}$ e $W_{i}$ são os pesos. Tal estimador produz errospadrão analíticos que são consistentes também na presença de heterocedasticidade.

No entanto, a literatura recente tem alertado para algumas limitações quanto ao uso do propensity score matching (PSM). King \& Nielsen (2016) mostram que da maneira usualmente utilizada, o PSM pode aumentar o desequilíbrio, a dependência do modelo, a discricionariedade da pesquisa e o viés estatístico dos dados reais, bem como dos próprios dados gerados para atender aos requisitos do PSM. Os autores citam que, em geral, os teoremas usados na literatura para justificar os escores de propensão, como em Rosenbaum \& Rubin (1983), não são relevantes para a prática do matching. Um desses problemas é que raramente o pesquisador conhece o processo gerador de dados, sendo necessário (sobretudo na análise de dados observacionais) descobrir em vez de projetar e implementar um processo gerador. Como existe uma vasta quantidade de modelos à escolha, o analista fica dependente do modelo, visto que dois ou mais métodos que ajustam os dados de forma aparentemente igual podem gerar estimativas causais empíricas diferentes. Para minimizar essa dependência, é comum encontrar nos trabalhos empíricos estimativas por diferentes métodos. Mesmo assim os autores citam que Ho et al. (2007) questionam se as demonstrações de diferentes estimativas não são apenas uma especificação das hipótese favorita do autor. Wilson \& Brekke (1994) relatam que existe uma tendência para imperceptivelmente favorecer as próprias hipóteses, o que é difícil de evitar. Nessas condições, os estimadores podem ser tendenciosos e viesados.

\footnotetext{
${ }^{21}$ São os agrupamentos de acordo com o status de tratamento.

${ }^{22}$ A seção de robustez será responsável por respaldar ou não tais estimativas.
} 
Outra limitação do PSM é que o algoritmo busca aproximar completamente de um experimento randomizado, ao contrário de outros métodos de correspondência que se aproximam de um experimento randomizado completamente bloqueado. Tal experimento bloqueado significa que o efeito de variáveis desnecessárias foi removido, tornando o matching mais eficiente. Em outras palavras, o PSM pode ser deficiente para a maioria do desequilíbrio que pode ser bloqueado por outros métodos de correspondência. É possível ainda que aumente o desequilíbrio e o viés do PSM quando se elimine as observações com o pior matching, de acordo com a distância absoluta do escore de propensão entre pares de tratados e não tratados, ao qual King \& Nielsen (2016) ilustram de paradoxo do PSM.

Porém quando o matching é bem sucedido, a dependência com relação ao modelo é reduzida. Para isso, existem várias técnicas de pareamento que podem ser usadas para reduzir a porcentagem do viés, como o Mahalanobis Distance Matching (MDM) e o Coarsened Exact Matching (CEM), que aproximam a um design experimental completo pois têm parâmetros ajustáveis para produzir o mesmo resultado que um matching exato, podendo ter um viés menor que o PSM. No entanto, o ideal seria ter um experimento randomizado totalmente aleatório, de modo a ter mais eficiência, maior robustez, menor dependência da escolha do método e menor viés.

O pesquisador também pode buscar reduzir o viés na aplicação do PSM, desde que observados alguns pontos. Em primeiro lugar, ele deve atentar para a escala das variáveis utilizadas de modo a representar sua importância, dado o conhecimento prévio sobre os efeitos de tais variáveis no outcome. Deve deixar claro ainda o algoritmo utilizado e o quanto da dependência e do viés foi deixado após a aplicação do PSM, para que o leitor saiba que não houve uma piora devido ao paradoxo do PSM. Outro ponto é que deve-se ter ciência que a aplicação do PSM é mais adequada para dados muito desequilibrados, nos quais inferências causais são menos prováveis. Por fim, é possível combinar o PSM com outros métodos de correspondência, usando o matching em um primeiro estágio e aplicando algum outro método no segundo estágio, comumente usado na literatura.

Outros estudos também chamam atenção para o uso incorreto do propensity score matching. Austin (2014), por exemplo, utiliza o PSM a partir de dois diferentes métodos de pareamento para replicar medidas de efeito frequentemente utilizadas em ensaios clínicos randomizados. Com base nos resultados, o autor desencoraja o uso de métodos de propensity score que resultem em estimativas condicionais de efeito de tratamento, pois pode resultar em superestimação dos benefícios do tratamento ou dos danos da exposição a um fenômeno.

Já o trabalho de Huber et al. (2013) investiga as propriedades em amostras finitas de uma série de estimadores (paramétricos e semiparamétricos) para o efeito de tratamento médio sobre os tratados ajustado para um vetor de variáveis observáveis, usando dados do mercado de trabalho para a Alemanha. A partir de diferentes variáveis de resultado, processos de seleção e tamanhos de amostra, os autores concluem que o estimador radius matching é o que apresenta melhor desempenho em relação ao erro quadrático médio, particularmente quando erros de especificação são considerados no propensity score. No entanto, todos os outros estimadores (que estão entre os melhores dentro de sua classe de estimadores) estão dentro de uma distância razoável em termos do erro quadrático médio. Por outro lado, Abadie \& Imbens (2016) propõem 
um método para corrigir a variância assintótica dos estimadores PSM para grandes amostras, levando em conta que o escore de propensão é estimado no primeiro estágio, antes do matching. Os resultados permitem uma inferência válida e mostram que sem a correção, a distribuição do PSM em grandes amostras pode ser afetada.

Dadas essas ressalvas, é preciso olhar com cautela para os resultados obtidos por estimadores PSM. Com isso, as próximas duas seções apresentam métodos de estimações robustos para contribuir com os achados a partir das estimativas desta seção. Antes será apresentado o método de correção do viés de seleção amostral, usado tanto para o estimador do efeito médio quanto para o estimador quantílico.

\section{Método de Correção do Viés de Seleção}

A renda do trabalho é observada somente para aqueles indivíduos que estão no mercado de trabalho. Tal fato conduz à autosseleção da amostra e à não consideração de características que fazem com que o indivíduo decida por ofertar trabalho, o que pode viesar as estimativas. Para obter a probabilidade de trabalhar ou não trabalhar existem diversos métodos, sendo o mais conhecido o Heckit, que usa um probit para calcular a inversa da razão de Mills e corrigir o viés de seleção (Heckman 1979).

No entanto, no presente trabalho será utilizado o método proposto por De Luca \& Perotti (2011) e aplicado por Oliveira (2014), que considera que a probabilidade de trabalhar ou não segue um processo não paramétrico. Dessa forma, Gallant \& Nychka (1987) demonstram que a densidade conjunta desconhecida pode ser aproximada por uma expansão polinomial de Hermite, sendo dada por

$$
f^{*}\left(u_{1}, u_{2} ; \gamma\right)=\frac{1}{\psi_{R}(\gamma)} \tau_{R}\left(u_{1}, u_{2} ; \gamma\right)^{2} \phi\left(u_{1}\right) \phi\left(u_{2}\right)
$$

em que $\gamma$ é um vetor dos parâmetros desconhecidos, $\tau_{R}\left(u_{1}, u_{2} ; \gamma\right)^{2}$ é um polinômio de ordem $R$ em $u_{1}$ e $u_{2}$, $\phi$ é uma função de densidade normal padrão e $\psi_{R}$ é uma normalização para garantir que $f^{*}$ seja uma função de densidade. Integrando a Equação 7, a função de densidade conjunta pode ser aproximada por

$$
\begin{gathered}
F^{*}\left(u_{1}, u_{2} ; \gamma\right)=\Phi\left(u_{1}\right) \Phi\left(u_{2}\right)+\frac{1}{\psi_{R}(\gamma)} A_{12}^{*}\left(u_{1}, u_{2} ; \gamma\right) \phi\left(u_{1}\right) \phi\left(u_{2}\right)- \\
\frac{1}{\psi_{R}(\gamma)} A_{1}^{*}\left(u_{1} ; \gamma\right) \Phi\left(u_{2}\right) \phi\left(u_{1}\right)-\frac{1}{\psi_{R}(\gamma)} A_{2}^{*}\left(u_{2} ; \gamma\right) \Phi\left(u_{1}\right) \phi\left(u_{2}\right),
\end{gathered}
$$

em que $A_{12}^{*}\left(u_{1}, u_{2} ; \gamma\right), A_{1}^{*}\left(u_{1} ; \gamma\right)$ e $A_{2}^{*}\left(u_{2} ; \gamma\right)$ são polinômios em $u_{1}$ e em $u_{2}$. As distribuições marginais de $u_{1}$ e $u_{2}$ podem ser encontradas de forma semelhante $^{23}$. Depois disso, o vetor de parâmetros é estimado de maneira semiparamétrica a partir da maximização da função de pseudoverossimilhança.

O vetor de variáveis explicativas incluído nessa etapa é formado, em especial, por covariadas sobre a decisão de oferta de trabalho, incluindo a renda do

\footnotetext{
${ }^{23}$ Para mais detalhes ver De Luca et al. (2008) e Oliveira (2014).
} 
não trabalho (aluguel, juros, pensão, aposentadoria, etc), características pessoais e familiares, hábitos de saúde, prática de exercícios físicos e dificuldades de mobilidade/locomoção. Todas as variáveis utilizadas nesse estágio estão também descritas na Tabela 4.

A variável dependente é a participação ou não no mercado de trabalho e, segundo Buchinsky (1998), deve ser estimada por um modelo semiparamétrico, por isso a escolha do modelo de resposta binária de estimadores de máxima verossimilhança semi não paramétricos ${ }^{24}$ (SNP) de Gallant \& Nychka (1987). Dessa etapa é obtida a inversa da razão de Mills, que junto com seu quadrado é inserida como variável explicativa na estimação do efeito de tratamento médio e quantílico, para avaliar a presença ou não de viés na seleção amostral.

\subsection{Identificação com Heterocedasticidade}

Dada a ausência de variáveis instrumentais externas que possam ser usadas diretamente nos modelos estimados na subseção anterior, a presente seção descreve o estimador de dois estágios proposto por Lewbel (2012), que explora a heterocedasticidade do primeiro estágio da regressão para gerar instrumentos internamente que possibilitem a identificação quando não existem restrições de exclusão disponíveis ${ }^{25}$.

Seguindo Lewbel (2012), o sistema triangular de equações como apresentado abaixo mostra que quando as correlações dos erros são causadas devido a fatores não observados, a identificação se dá tendo regressores não correlacionados com o produto dos erros heterocedásticos.

Para o propósito deste artigo, vamos supor $U$ como sendo uma variável omitida que possivelmente afeta a variável endógena de tratamento "estado de saúde"(denotada por $S$ ) tanto quanto afeta o resultado renda do trabalho $Y$, e deixando $V_{1}$ e $V_{2}$ como sendo os erros idiossincráticos. Portanto, é possível identificar o efeito causal das condições de saúde em $Y$, denotado por $\beta$, por meio do Método dos Momentos Generalizados (GMM) ou de Mínimos Quadrados Modificados em Dois Estágios (2SLS).

$$
\begin{gathered}
Y=X^{\prime} \beta_{1}+\beta S+\varepsilon_{1} \\
S=X^{\prime} \beta_{2}+\varepsilon_{2} \\
E\left[X \varepsilon_{1}\right]=0, E\left[X \varepsilon_{2}\right]=0, \operatorname{Cov}\left[Z, \varepsilon_{1} \varepsilon_{2}\right]=0,
\end{gathered}
$$

Em que $\varepsilon_{1}=\alpha_{1} U+V_{1}$ e $\varepsilon_{2}=\alpha_{2} U+V_{2}$ e $Z \subseteq \mathrm{X}^{26}$. A Equação 11 apresenta as condições necessárias para a identificação e estimação, juntamente com alguma heterocedasticidade em $\varepsilon_{i}{ }^{27}$. Assim, a classe de modelos que satisfazem os pressupostos subjacentes ao método de Lewbel são aqueles que as correlações dos erros nas equações em cross-section são por conta da presença de um fator comum não observado.

\footnotetext{
${ }^{24} \mathrm{O}$ comando semi-nonparametric (SNP) desenvolvido por De Luca et al. (2008) é usado para a estimação desses coeficientes.

${ }^{25} \mathrm{O}$ método pode ser usado também para melhorar a eficiência do estimador IV padrão.

${ }^{26}$ Note que $Z$ é um subconjunto de $X$, e com isso, nenhuma informação de fora do modelo especificado é necessária.

${ }^{27}$ Para mais detalhes ver Lewbel (2012) e Baum \& Schaffer (2012).
} 
A equação auxiliar ou regressão do primeiro estágio pode ser usada para fornecer os elementos necessários para o método de Lewbel. Em sua versão mais simples, instrumentos podem ser construídos a partir dos resíduos das regressões auxiliares multiplicado por cada uma das variáveis exógenas centrada na média, ou seja:

$$
Z_{j}=\left(X_{j}-\bar{X}\right) \cdot \epsilon
$$

Em que $\epsilon$ é o vetor de resíduos da regressão do primeiro estágio de cada regressor endógeno contra todos os regressores exógenos, incluindo um vetor de constantes. Esses resíduos têm covariância zero com cada um dos regressores usados para construí-los, o que implica que a média dos instrumentos gerados são zero. Por outro lado, o produto desses resíduos com os elementos dos regressores centrados na média não será zero se houver evidência considerável de heterocedasticidade de escala com relação às variáveis explicativas ${ }^{28}$. Assim, quanto maior o grau da heterocedasticidade no processo de erro, maior será a correlação dos instrumentos gerados com as variáveis endógenas incluídas (que são os regressandos das regressões auxiliares).

É claro que o método fornece estimativas menos confiáveis do que se tivesse uma variável instrumental exógena para ser usada nas estimativas. No entanto, alguns trabalhos empíricos como Emran \& Hou (2013), Amorim \& Sampaio (2015) e Tigre et al. (2017) têm mostrado que a abordagem de Lewbel produz resultados muito parecidos quando comparado com a estimativa obtida com o uso de instrumentos tradicionais.

Em suma, na falta de uma variável instrumental externa válida, como é o caso de muitas aplicações empíricas, o método de Lewbel é uma alternativa interessante para estimar o efeito causal de interesse, nesse caso, do estado de saúde sobre o salário por hora dos indivíduos.

\subsection{Bounding para o Efeito de Tratamento}

Como último procedimento empírico adotado no presente trabalho, esta seção apresenta o método recentemente proposto por (Oster 2013, 2015) para estimar bounds para o efeito de tratamento, usado para corroborar os resultados encontrados com as estratégias das subseções anteriores.

A ideia é que os movimentos no coeficiente de interesse, nesse caso, a condição de saúde individual, carrega informação sobre o viés remanescente devido a variáveis não observadas, dada a inclusão ou não de controles devido à preocupação com variáveis omitidas. Em outras palavras, o método segue a noção de Altonji et al. (2005) de que as variáveis não observáveis não devem ser mais importantes do que as observáveis na explicação do tratamento.

Para descrever o método, considere que existe um conjunto de variáveis não observáveis, $U$, tal como:

$$
Y=\alpha+\beta S+X^{\prime} \gamma+U+\varepsilon
$$

O pressuposto de seleção proporcional afirma que $\delta \frac{\sigma_{X S}}{\sigma_{X}}=\frac{\sigma_{U S}}{\sigma_{U}}$, em que $\sigma_{X S}=\operatorname{Cov}(X, S), \sigma_{U S}=\operatorname{Cov}(U, S), \sigma_{X}=\operatorname{Var}(X)$ e $\sigma_{U}=\operatorname{Var}(U)$, e o coeficiente de proporcionalidade, $\delta$, é informativo sobre como as não observáveis se relacionam com o tratamento, dado que é conhecido como as observáveis se

\footnotetext{
${ }^{28} \mathrm{~A}$ heterocedasticidade pode ser analisada por testes tal como o de Breusch-Pagan.
} 
relacionam com tal tratamento. Dessa forma, se $\delta=1$, por exemplo, a seleção em não observáveis é igual à seleção sobre as observáveis.

Dito isso, considere as equações a seguir, em que o $R$-quadrado do modelo completo representado pela Equação 14 é o $R_{\max }$, e para as Equações 15 e 16 é $R_{1}$ e $R_{2}$, respectivamente. A Equação 16 inclui $M$, que é um conjunto restrito de controles observados e não tem um componente não observado associado, além de ser ortogonal com $X$ e $U$.

$$
\begin{gathered}
Y=\alpha+\beta S+X^{\prime} \gamma+U+\varepsilon_{\text {max }} \\
Y=\alpha+\beta_{1} S+X^{\prime} \gamma+\varepsilon_{1} \\
Y=\alpha+\beta_{2} S+M^{\prime} \alpha+\varepsilon_{2}
\end{gathered}
$$

Sob essas restrições, e quando $\delta$ é próximo de 1 , o valor de $B(\delta)=\delta \frac{\left(\beta_{2}-\beta_{1}\right)\left(R_{\max }-R_{1}\right)}{R_{1}-R_{2}}$ é igual a:

a) O viés não observado se $\delta=1$;

b) Próximo ao limite superior do viés se $\delta<1$;

c) Próximo ao limite inferior do viés se $\delta>1$;

Observe que o vetor de controles observados $X$ é uma seleção aleatória do conjunto total $(X, U)$, quando $\delta=1$. Oster (2013) argumenta que as covariáveis mais importantes para explicar o tratamento geralmente estão contidas no vetor $X$, o que implica que $\delta$ deve ser maior que um. Em outras palavras, a seleção em não observáveis não deve exceder a seleção sobre as observáveis.

Portanto, dada essa configuração, é possível obter estimativas não viesadas para o efeito de tratamento, calculando o viés via $B(\delta)$, que para seu cálculo é necessário conhecer o valor de $R_{\max }$, que não é observado. No entanto, deve haver alguma aleatoriedade nos movimentos da variável de resultado fazendo com que o $R_{\max }$ seja menor que 1 . Nas estimações desse presente trabalho, foram considerados valores para o $R_{\max }$ variando de $0,7^{29}$ até 1 , sendo este último um valor muito prudente, usado para o cálculo de $\delta^{30}$.

De acordo com a abordagem efetuada em Altonji et al. (2005) e Oster (2013), é possível obter um valor de $\delta$ que seria suficiente para explicar o efeito de tratamento por completo, isto é, que faria com que $\hat{\beta}=0$. Esse valor daria uma ideia do grau de seleção nas não observáveis relativo às observáveis, que seria necessário para o tratamento ser completamente explicado por variáveis não observadas, não incluídas no modelo.

\section{Resultados}

Esta seção apresenta o efeito das condições de saúde sobre os rendimentos do trabalho com base no propensity score para o efeito de tratamento médio e o efeito de tratamento quantílico, usando características pessoais, de localização, de ramo de atividade e status de trabalho como controles. Na Subseção 5.1, serão apresentados os resultados baseados nos métodos de Lewbel (2012) e Oster (2015) como robustez para as estimativas aqui apresentadas.

\footnotetext{
${ }^{29}$ Usado em Oster (2013).

${ }^{30}$ Também usado em Oliveira et al. (2015). Para mais detalhes ver Oster (2013).
} 
Antes disso, a Tabela 5 apresenta as variáveis que explicam as diferenças de probabilidade de ter uma má condição de saúde autorreportada ou ser diagnosticado com alguma doença por um profissional, por meio de um propensity score logístico. Foram incluídas todas as características observadas que podem afetar tanto o tratamento (condição de saúde) quanto o resultado (renda do trabalho). É importante relembrar que as variáveis binárias das condições de saúde são construídas de modo a assumir o valor 1 para uma má condição autorreportada (Autoavaliação de Saúde) e para ter sido diagnosticado com alguma doença crônica (doenças). Dessa forma, coeficientes positivos indicam que a variável em questão impacta para aumentar a péssima condição de saúde, enquanto que coeficientes negativos impactam para reduzir a má condição de saúde.

Apesar de uma diferença no valor dos coeficientes entre as duas variáveis de saúde, ser do sexo feminino e casado afetam a probabilidade de ter uma má condição de saúde. Como visto na Seção 3.1, um maior percentual de mulheres é diagnosticado com praticamente todas as doenças listadas em relação aos homens, assim como uma maior proporção relata uma má condição de saúde autoavaliada. Tal resultado segue em linha com Gomes et al. (2014), e pode ser explicado pelo fato de as mulheres fazerem mais consultas médicas (inclusive exames de rotina e prevenção) e com isso conhecerem melhor sua real condição.

Outras variáveis que contribuem para uma maior probabilidade de saúde insatisfatória é o aumento da idade, que naturalmente debilita a saúde individual, e o tamanho da família, que faz com que se tenha menos recurso per capita para cuidados com a saúde. Ser fumante e residir em área urbana também aumentam a probabilidade de não ser saudável, esta última possivelmente pelo estilo de vida mais estressante levado nas cidades, apesar do maior acesso aos serviços de saúde.

Por outro lado, indivíduos brancos e que praticam atividade física reduzem a probabilidade de reportar uma condição não saudável, sendo que a raça pode estar ligada ao diferencial de rendimentos em relação aos não brancos, fato bastante consolidado na literatura sobre discriminação salarial (ver por exemplo Cacciamali \& Hirata (2005), e Coelho et al. (2010)). O nível de escolaridade também contribui para uma menor probabilidade de má saúde, resultado consolidado na literatura que mostra uma relação positiva entre educação e status de saúde, tal como em Sousa et al. (2010).

A relação entre educação e saúde pode se dar diretamente ou a partir dos efeitos da renda. De maneira direta, pessoas mais escolarizadas tendem a adotar hábitos de vida mais saudáveis e a procurar mais os serviços médicos, especialmente os cuidados preventivos, uma vez que, ao possuírem mais informações sobre as doenças e os possíveis tratamentos ou diagnósticos, tendem a valorizar de forma diferenciada o cuidado com a saúde. Com relação à renda, esta tende a aumentar com o nível de escolaridade, de modo que o indivíduo dispõe de melhores condições de vida e de acesso aos serviços de saúde.

Por outro lado, é preciso atentar para a possibilidade de o efeito se dar também no sentido inverso, dado que uma melhor condição de saúde torna os indivíduos mais dispostos e aptos a realizar diversas atividades (inclusive estudar) e com isso gerar mais renda. Além disso, como se observa apenas um ponto no tempo, não é possível saber o estoque inicial de saúde ou rendimento dos indivíduos, o que facilitaria em estabelecer uma relação causal mais confiável. 
Tabela 5: Matching logit - regressão do tratamento em todas as outras variáveis

\begin{tabular}{|c|c|c|}
\hline & \multicolumn{2}{|c|}{ Tratamento } \\
\hline Variáveis & $\begin{array}{c}\text { Auto Avaliação } \\
\text { de Saúde }\end{array}$ & Doenças $^{a}$ \\
\hline Sexo & $\begin{array}{l}0,3477^{* * *} \\
(0,0253)\end{array}$ & $\begin{array}{l}0,5311^{* * * *} \\
(0,0216)\end{array}$ \\
\hline Raça & $\begin{array}{l}-0,2143^{* * *} \\
(0,0231)\end{array}$ & $\begin{array}{l}-0,0627^{* * *} \\
(0,0201)\end{array}$ \\
\hline Estado Civil & $\begin{array}{l}0,1162^{* * *} \\
(0,0256)\end{array}$ & $\begin{array}{l}0,0901^{* * *} \\
(0,0224)\end{array}$ \\
\hline Idade & $\begin{array}{l}0,0380^{* * *} \\
(0,0010)\end{array}$ & $\begin{array}{l}0,0599^{* * *} \\
(0,0009)\end{array}$ \\
\hline Ano de Estudo & $\begin{array}{l}-0,0815^{* * *} \\
(0,0029)\end{array}$ & $\begin{array}{l}-0,0259^{* * *} \\
(0,0025)\end{array}$ \\
\hline Fumante & $\begin{array}{l}0,0523^{* *} \\
(0,0251)\end{array}$ & $\begin{array}{l}0,0649^{* * *} \\
(0,0226)\end{array}$ \\
\hline Atividade Física & $\begin{array}{l}-0,3659^{* * *} \\
(0,0257)\end{array}$ & $\begin{array}{l}-0,0880^{* * *} \\
(0,0210)\end{array}$ \\
\hline Renda (Não Trabalho) ${ }^{1}$ & $\begin{array}{r}-0,0001 \\
(0,0001)\end{array}$ & $\begin{array}{r}-0,0001 \\
(0,0001)\end{array}$ \\
\hline Tamanho da Família & $\begin{array}{l}0,0243^{* * * *} \\
(0,0074)\end{array}$ & $\begin{array}{r}-0,0115^{*} \\
(0,0067)\end{array}$ \\
\hline Militar $^{2}$ & $\begin{array}{c}-0,0095 \\
(0,0518)\end{array}$ & $\begin{array}{l}0,1137^{* * *} \\
(0,0428)\end{array}$ \\
\hline Com Carteira & $\begin{array}{l}-0,1389^{* * *} \\
(0,0343)\end{array}$ & $\begin{array}{c}0,0267 \\
(0,0299)\end{array}$ \\
\hline Doméstico & $\begin{array}{c}0,2720^{*} \\
(0,1407)\end{array}$ & $\begin{array}{c}0,0127 \\
(0,1117)\end{array}$ \\
\hline Conta - Própria & $\begin{array}{l}0,1250^{* * *} \\
(0,0331)\end{array}$ & $\begin{array}{l}0,1296^{* * * *} \\
(0,0302)\end{array}$ \\
\hline Empregador & $\begin{array}{l}-0,2457^{* * *} \\
(0,0568)\end{array}$ & $\begin{array}{l}0,1309^{* * *} \\
(0,0463)\end{array}$ \\
\hline Urbana & $\begin{array}{c}0,0461 \\
(0,0353)\end{array}$ & $\begin{array}{l}0,1257^{* * * *} \\
(0,0330)\end{array}$ \\
\hline Constante & $\begin{array}{l}5,6249^{* * *} \\
(0,3582)\end{array}$ & $\begin{array}{c}4,7451^{* * *} \\
(0,4025)\end{array}$ \\
\hline $\begin{array}{l}\text { Ocupação (FE) } \\
\text { UF (FE) } \\
\text { Observações } \\
\text { Log Likelihood }\end{array}$ & $\begin{array}{c}\text { Sim } \\
\text { Sim } \\
60.812 \\
-38.029,045\end{array}$ & $\begin{array}{c}\text { Sim } \\
\text { Sim } \\
60.812 \\
-45.886,874\end{array}$ \\
\hline
\end{tabular}

Fonte: Elaboração própria a partir dos dados das PNAD de 2008. Notas: Desvios-padrão entre parênteses. ${ }^{* * *}$ p-valor $<0,01 .{ }^{* *} \mathrm{p}$-valor $<0,05 .{ }^{*} \mathrm{p}$-valor $<0,10$.

a. Ter pelo menos uma doença constatada por um médico.

1. Soma de todos os rendimentos do não trabalho (pensão, aposentadorias, juros, aluguel, etc);

2. Categoria base: sem carteira. 
Até esse ponto, os resultados coincidem para as duas variáveis de saúde. No entanto, para o ramo de atividade, algumas características têm impactos contrários, destacando o fato de ser empregador, que reduz a probabilidade da autopercepção de má saúde e aumenta a probabilidade de ter alguma doença diagnosticada. Isso pode ser devido à carga de trabalho, que acarreta alguma doença crônica diagnosticada. Por fim, ainda foram incluídas dummies de efeito fixo para cada um dos Estados e para o tipo de ocupação no mercado de trabalho.

A Tabela 6 abaixo apresenta o efeito médio com a correção do viés de seleção amostral para diferentes condições de saúde usadas como tratamento. Além da condição autoavaliada e do diagnóstico de pelo menos uma doença, também são usados o diagnóstico de duas ou mais doenças e de três ou mais doenças, como já explicitado antes, sendo o grupo de controle o indivíduo saudável em ambos os $\operatorname{casos}^{31}$.

O efeito é negativo em todas as especificações, evidenciando uma redução de rendimentos do trabalho devido a uma condição de saúde insatisfatória. $\mathrm{O}$ impacto da autoavaliação de saúde é maior que o impacto do diagnóstico de uma doença, o qual, mesmo sendo um critério subjetivo, pode estar mensurando de forma mais adequada o estado de saúde e evidencia que indivíduos que afirmam ter uma má condição de saúde autorreportada tendem a ter um menor desempenho e produtividade, comparado ao diagnóstico de uma doença.

À medida que o número de doenças usado como tratamento aumenta, é observada uma elevação na magnitude do coeficiente, indicando uma deterioração da saúde e consequentemente da capacidade produtiva individual, refletindo-se assim no aumento da perda de rendimento do trabalho.

Tabela 6: Estimação do efeito de tratamento médio

\begin{tabular}{|c|c|c|c|c|}
\hline \multirow[b]{2}{*}{ Método } & \multicolumn{4}{|c|}{ Tratamento } \\
\hline & Autoavaliação & $\begin{array}{c}\text { Doenças } \\
(1 \text { doença })\end{array}$ & $\begin{array}{c}\text { Doenças } \\
(2 \text { ou mais })\end{array}$ & $\begin{array}{c}\text { Doenças } \\
\text { (3 ou mais) }\end{array}$ \\
\hline ATT & $\begin{array}{c}-0,117^{* * *} \\
(0,006)\end{array}$ & $\begin{array}{c}-0,042^{* * *} \\
(0,013)\end{array}$ & $\begin{array}{c}-0,356^{* * *} \\
(0,019)\end{array}$ & $\begin{array}{c}-0,799^{* * *} \\
(0,050)\end{array}$ \\
\hline Controles & Sim & Sim & Sim & Sim \\
\hline Ocupação (FE) & Sim & Sim & Sim & Sim \\
\hline $\mathrm{UF}(\mathrm{FE})$ & Sim & Sim & Sim & Sim \\
\hline Observações & 60.832 & 60.832 & 46.397 & 40.735 \\
\hline
\end{tabular}

No entanto, é preciso ter cautela com os resultados apresentados na Tabela 6 , tendo em vista as ressalvas feitas quanto ao uso do PSM na seção metodológica. Conforme mencionado anteriormente, existe também a possibilidade de a relação entre condição de saúde e renda ser simultânea. Outra limitação reside nos dados serem em cross-section, não se tendo informações sobre o esto-

\footnotetext{
${ }^{31}$ Por exemplo, na construção da dummy que indica ter duas ou mais doenças $=1$ e nenhuma doença $=0$ os indivíduos que apresentam apenas uma doença são excluídos. O mesmo é feito para a dummy que indica ser diagnosticado com três ou mais doenças.
} 
que de saúde ou de entrada no mercado de trabalho dos indivíduos, tornando a causalidade mais difícil de ser encontrada.

Porém, Smith (1999) destaca que o efeito da renda na saúde é mais pronunciado em indivíduos jovens, enquanto em idades mais avançadas o efeito da condição de saúde na renda tende a ser maior. Como a amostra é composta por adultos (ver na Tabela 4 que a média de idade é de 40 anos), é razoável assumir que estes já possuem um estoque de saúde acumulado em etapas anteriores de suas vidas, que terá um efeito na renda das etapas posteriores. É claro que uma baixa renda em períodos anteriores acarreta em menos cuidados com a saúde, ao passo que uma pior condição de saúde vai permitir menor capacidade para acumulação de renda futura, gerando um ciclo vicioso que se perpetua no tempo. No entanto, quando se controla por características observáveis, ainda podemos ter indícios de que indivíduos com um status de saúde deteriorado podem ter reflexos negativos no mercado de trabalho.

Esse resultado caminha no mesmo sentido das evidências empíricas presentes na literatura que mostram um efeito negativo da condição de saúde no rendimento, nas horas trabalhadas ou até mesmo na exclusão do mercado de trabalho. No entanto, foram utilizados aqui vários tratamentos para evidenciar a importância da saúde autoavaliada como reconhecimento do indivíduo acerca de sua capacidade e ainda um aumento do efeito quando cresce o número de doenças diagnosticadas no indivíduo.

Na Tabela 7, foi utilizada cada uma das doze doenças listadas na seção de dados como tratamento individual, controlado por todas as demais características supracitadas, inclusive pelas demais doenças. Com exceção do câncer e cirrose, que apesar de apresentarem um coeficiente positivo não foram estatisticamente significativos, todas as demais doenças impactaram negativamente o salário por hora dos trabalhadores, chegando a -0,209 o efeito da doença renal.

Para verificar o impacto ao longo de toda a distribuição de rendimentos, a Figura 1 sintetiza os resultados do efeito de tratamento quantílico corrigido pelo viés de seleção para cada um dos quatro tratamentos utilizados. Diferente da estimação pela média condicional da variável dependente, esse método permite visualizar como os efeitos do tratamento se alteram em diferentes quantis.

São plotadas na Figura as estimativas do coeficiente da dummy de condição de saúde para cada quantil entre 0,05 e 0,95, bem como o intervalo de confiança de $95 \%$, representado pela área cinza em volta da curva. Assim como no efeito médio, a redução analisada pela autoavaliação de saúde é maior que o diagnóstico de uma doença ao longo de toda a distribuição, corroborando a ideia de um indicador global da real condição de saúde para este primeiro. $\mathrm{O}$ impacto maior da autoavaliação de saúde se dá nos dois extremos da distribuição, o que pode indicar uma má percepção pela condição de pobreza nos quantis inferiores, e uma carga excessiva de trabalho nos quantis superiores. Com relação ao diagnóstico de uma doença, os coeficientes mantêm certa estabilidade ao longo da distribuição, sendo o efeito negativo, mas próximo de zero.

Os coeficientes ao longo de toda a distribuição para o tratamento duas ou mais doenças e três ou mais doenças se mostraram menores que aquele do efeito médio, o que sugere evidências de uma superestimação do impacto na média. Além disso, os trabalhadores localizados nos menores quantis tiveram uma redução de salário muito maior que os quantis superiores, corroborando 
Tabela 7: Efeito de tratamento médio. Tratamento - cada doença individualmente

\begin{tabular}{lc}
\hline Tratamento & Estimação ATT \\
\hline \multirow{3}{*}{ Coluna } & Coeficiente \\
\cline { 2 - 2 } Artrite & $-0,047^{* * *}$ \\
& $(0,003)$ \\
Câncer & $-0,080^{* * *}$ \\
& $(0,001)$ \\
Diabetes & 0,029 \\
& $(0,097)$ \\
Bronquite & $-0,014^{* * *}$ \\
& $(0,033)$ \\
Hipertensão & $-0,031^{* * *}$ \\
& $(0,041)$ \\
Coração & $-0,031^{* * *}$ \\
& $(0,004)$ \\
Doença Renal & $-0,061^{* * *}$ \\
& $(0,029)$ \\
Depressão & $-0,209^{* * *}$ \\
& $(0,054)$ \\
Tuberculose & $-0,128^{* * *}$ \\
& $(0,006)$ \\
Tendinite & $-0,079^{* * *}$ \\
& $(0,026)$ \\
Cirrose & $0,099^{* * *}$ \\
& $(0,027)$ \\
\hline Fonte: Elaboração própria a partir dos \\
dados das PNAD de 2008. Notas: \\
Desvios-padrão robustos a \\
heterocedasticidade entre parênteses. \\
$* * *$ p-valor $<0,01 .{ }^{* *}$ p-valor $<0,05 .{ }^{*}$ \\
p-valor $<0,10$ & 0,048 \\
& $(0,178)$ \\
&
\end{tabular}

a ideia do círculo vicioso como visto em Gomes et al. (2014). Isso significa que os trabalhadores que têm menores rendimentos são justamente aqueles que têm a maior perda quando se encontram doentes, o que pode torná-los mais pobres e agravar mais ainda a condição de saúde. Além disso, os trabalhadores com menores rendimentos são aqueles que tendem a ocupar trabalhos que demandam maior esforço físico, ficando muitas vezes impossibilitado de exercer suas tarefas com alguma enfermidade.

O efeito negativo vai se tornando menor ao longo da distribuição chegando a ser praticamente zero para os indivíduos que recebem os mais altos rendimentos. Em suma, o efeito de uma má condição de saúde é menor para aqueles indivíduos que têm maiores rendimentos, o que implica uma maior capacidade de adaptação desses trabalhadores ao mercado de trabalho, levando em conta a gravidade da enfermidade e o fato de que, em geral, ocupam trabalhos que exigem menos esforço físico, sendo possível conviver com tal condição. 
Tais resultados corroboram com a possível simultaneidade da relação, conforme já mencionado. O efeito maior para os indivíduos nos quantis inferiores pode se dever ao fato de que estes já tenham trabalhos menos qualificados por possuírem um menor estoque de saúde acumulado ao longo do tempo, o que reduz os cuidados e recursos disponíveis com a saúde. Se esses indivíduos tiveram uma pior entrada no mercado de trabalho (ocupações menos qualificadas e com baixa remuneração), terão menos recursos para investir em saúde e consequentemente uma menor capacidade de gerar renda.

Os dados analisados em um ponto no tempo não permitem identificar as condições iniciais de saúde e nem o efeito de um emprego não qualificado nesta. No entanto, a literatura e os achados até aqui têm evidenciado que os efeitos podem persistir ao longo do tempo, e uma má condição de saúde tem impacto negativo no rendimento do trabalho quando os indivíduos não conseguem desenvolver completamente suas atividades, afetando sua produtividade. Como já mencionado, os resultados desta seção são apenas preliminares, dadas as limitações nos dados e questões envolvendo causalidade reversa que podem estar presentes. As próximas subseções buscam corroborar e dar maior robustez a esses achados, bem como inferir com maior confiabilidade o impacto da condição de saúde no rendimento do trabalho.

Dessa forma, o teste de robustez com a estimação do impacto das condições de saúde usando a heterocedasticidade para gerar instrumentos internos e bounds para o efeito do tratamento ajudará a confirmar o efeito negativo de tais condições sobre os rendimentos do trabalho.

\subsection{Análise de Robustez}

As evidências mostram o impacto negativo de uma má condição de saúde na renda do trabalho individual. Esta seção começa pela apresentação dos resultados do efeito de tratamento médio estimado por Generalized Method of Moments (GMM) a partir do método de Lewbel (2012), que explora a identificação a partir da heterocedasticidade dos resíduos para gerar instrumentos internos, quando o produto de tais erros não é correlacionado com os regressores $^{32}$.

As estimativas corroboram os resultados anteriores de um efeito negativo da condição de saúde sobre o rendimento dos trabalhadores, independente do tratamento utilizado. No entanto, tal como no efeito quantílico, o método de Lewbel evidencia uma possível superestimação do efeito médio quando não considerados os instrumentos para a condição de saúde. Tal fato pode ser visto pela redução de todos os coeficientes, como, por exemplo, da saúde autoavaliada como tratamento, que passa de $-0,117$ (efeito de tratamento médio) para -0,0619 (efeito médio pelo método de Lewbel). Para duas/três ou mais doenças como tratamento, a redução do coeficiente é ainda maior.

É importante frisar que a utilização de instrumentos internos pelo método de Lewbel ajuda a contornar o problema da causalidade reversa, pois buscamos captar aquela parte da condição de saúde que afeta o rendimento, mas não é afetada por este. Mesmo sem conhecer o estoque de saúde dos indivíduos, os resultados apontam a possibilidade de um efeito negativo na renda do trabalho, dado um nível de saúde autoreportado como insatisfatório ou tendo sido diagnosticado com alguma doença.

\footnotetext{
${ }^{32}$ Foi realizado o teste de White (1980) e rejeitada a hipótese nula de homocedasticiade (verificada a presença de heterocedasticiade), condição para a validade do método.
} 
Figura 1: Coeficientes da regressão quantílica - PNAD - 2008

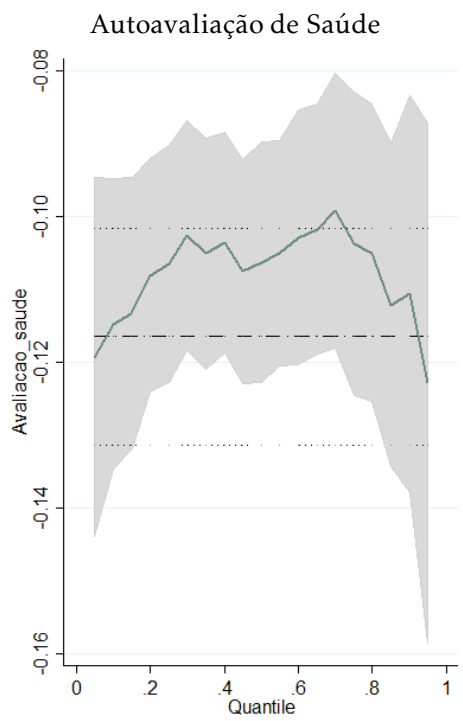

Doenças (1 doença)

Doenças (2 ou mais)

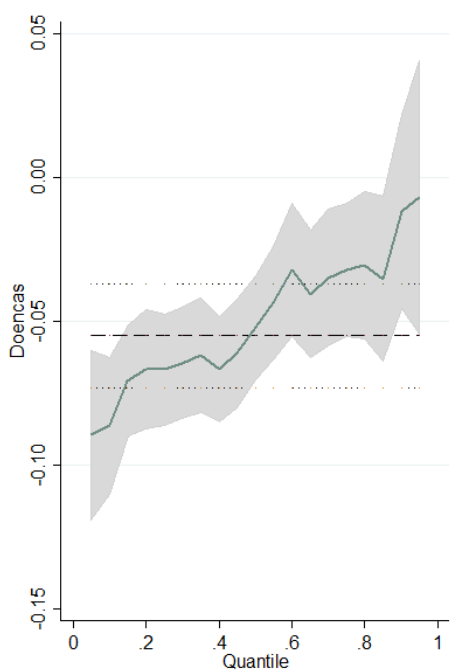

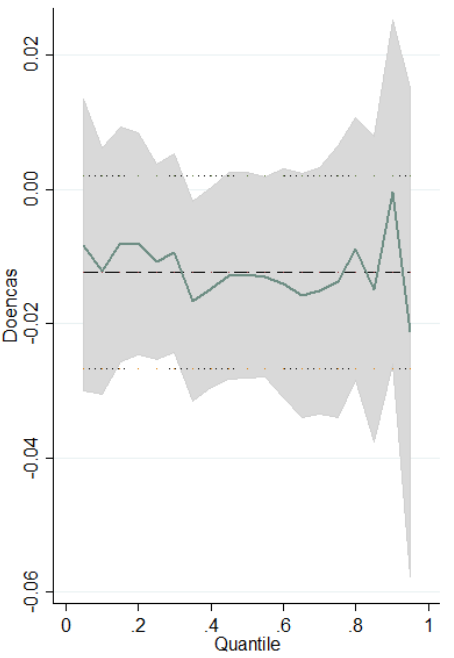

Doenças (3 ou mais)

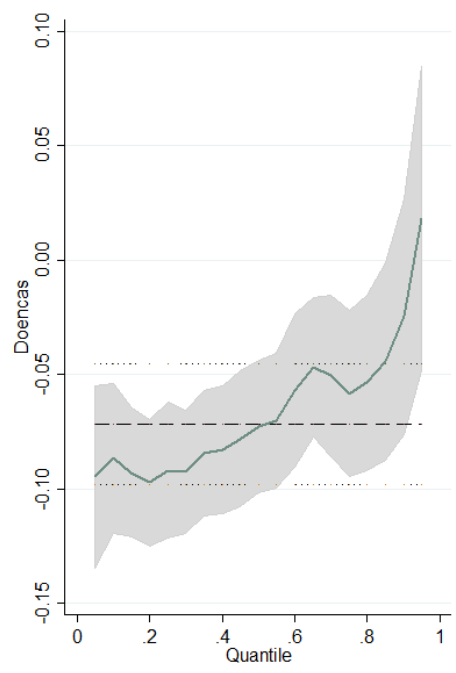

Fonte: Elaboração própria com base nas estimações. 
Tabela 8: ATT estimado usando o método de Lewbel (2012)

\begin{tabular}{lcccc}
\hline \multirow{2}{*}{ Método } & \multicolumn{4}{c}{ Tratamento } \\
\cline { 2 - 5 } & Autoavaliação & $\begin{array}{c}\text { Doenças } \\
(1 \text { doença) }\end{array}$ & $\begin{array}{c}\text { Doenças } \\
(2 \text { ou mais })\end{array}$ & $\begin{array}{c}\text { Doenças } \\
(3 \text { ou mais })\end{array}$ \\
\hline$A T T_{G M M}$ & $-0,0619^{* * *}$ & $-0,0318^{* *}$ & $-0,0720^{* * *}$ & $-0,1070^{* *}$ \\
& $(0,0175)$ & $(0,0190)$ & $(0,0161)$ & $(0,0464)$ \\
Controles & $\mathrm{Sim}$ & $\mathrm{Sim}$ & $\mathrm{Sim}$ & $\mathrm{Sim}$ \\
Ocupação $(\mathrm{FE})$ & $\mathrm{Sim}$ & $\mathrm{Sim}$ & $\mathrm{Sim}$ & $\mathrm{Sim}$ \\
UF (FE) & $\mathrm{Sim}$ & $\mathrm{Sim}$ & $\mathrm{Sim}$ & $\mathrm{Sim}$ \\
\hline Observações & 60.548 & 60.548 & 46.174 & 40.531 \\
K-P LM & $4017.766^{* * *}$ & $47.591^{* * *}$ & $95.640^{* * *}$ & 1497.596
\end{tabular}

Fonte: Elaboração própria a partir dos dados das PNAD de 2008.

Notas: Desvios-padrão robustos a heterocedasticidade entre parênteses, com bootstrap de 1000 replicações. ${ }^{* *}$ p-valor $<0,01 .{ }^{* *}$ p-valor $<0,05 .{ }^{*}$ p-valor $<0,10$

A última linha da Tabela 8 apresenta o teste de subidentificação do modelo, no qual se rejeita a hipótese nula indicando que o modelo é identificado. Já o teste de Cragg-Donald rejeita a hipótese nula de fracos instrumentos, sugerindo que os instrumentos internos gerados são relevantes para o tratamento $^{33}$. O artigo de Emran \& Hou (2013) encontra uma semelhança qualitativa entre as estimativas obtidas por instrumentos externos e pelos gerados pelo presente método, de modo que, apesar de não dispor de variáveis de exclusão externas, é possível ter certo grau de confiança nos instrumentos gerados para o presente trabalho.

Por fim, são estimados os bounds para o efeito de tratamento da condição de saúde seguindo Oster (2015) para obter os valores da seleção nas não observáveis, que seriam necessários para explicar completamente as estimativas, em comparação com a seleção nas observáveis. Para cada variável de tratamento, são considerados quatro valores de $R_{\max }$ para se obter o valor do coeficiente de proporcionalidade $(\delta)$ que seria necessário para fazer a estimativa do efeito de tratamento ir para zero $(\beta=0)$ e também para se obter um limite inferior para o efeito (o conjunto identificado).

A Tabela 9 apresenta tais resultados para cada condição de saúde usada como tratamento. Para a autoavaliação de saúde, considerando o $R_{\max }$ de 0,7 , por exemplo, foi encontrado um coeficiente de proporcionalidade de 2,8034, o que implica que o efeito das variáveis não observadas no ATT estimado ao longo da condição de saúde teria que ser 2,8034 vezes mais forte do que o efeito das variáveis observáveis para explicar todo o efeito negativo da condição de saúde no rendimento do trabalho. Em outras palavras, as não observáveis teriam que ser 2,8034 vezes mais importantes na explicação do efeito médio, de modo a fazer com que o coeficiente baseado nas observáveis fosse zero. Aumentando o $R_{\max }$ para um valor muito improvável de 1 , tal coeficiente ainda é de 1,4694. Para o limite inferior, o valor é de $-0,20$ (para $R_{\max }=$ $0,7)$ e passa para $-0,09$ (para $R_{\max }=1,0$ ).

Para as demais variáveis de tratamento a interpretação é semelhante, o que corrobora os achados das tabelas anteriores, qual seja, de que o viés causado pelas não observáveis não inviabiliza os coeficientes estimados. Oster (2015), analisando o impacto do comportamento materno sobre as crianças, encon-

\footnotetext{
${ }^{33}$ Os resultados do teste podem ser solicitados junto aos autores.
} 
Tabela 9: Bounds para o efeito do tratamento - Oster (2015)

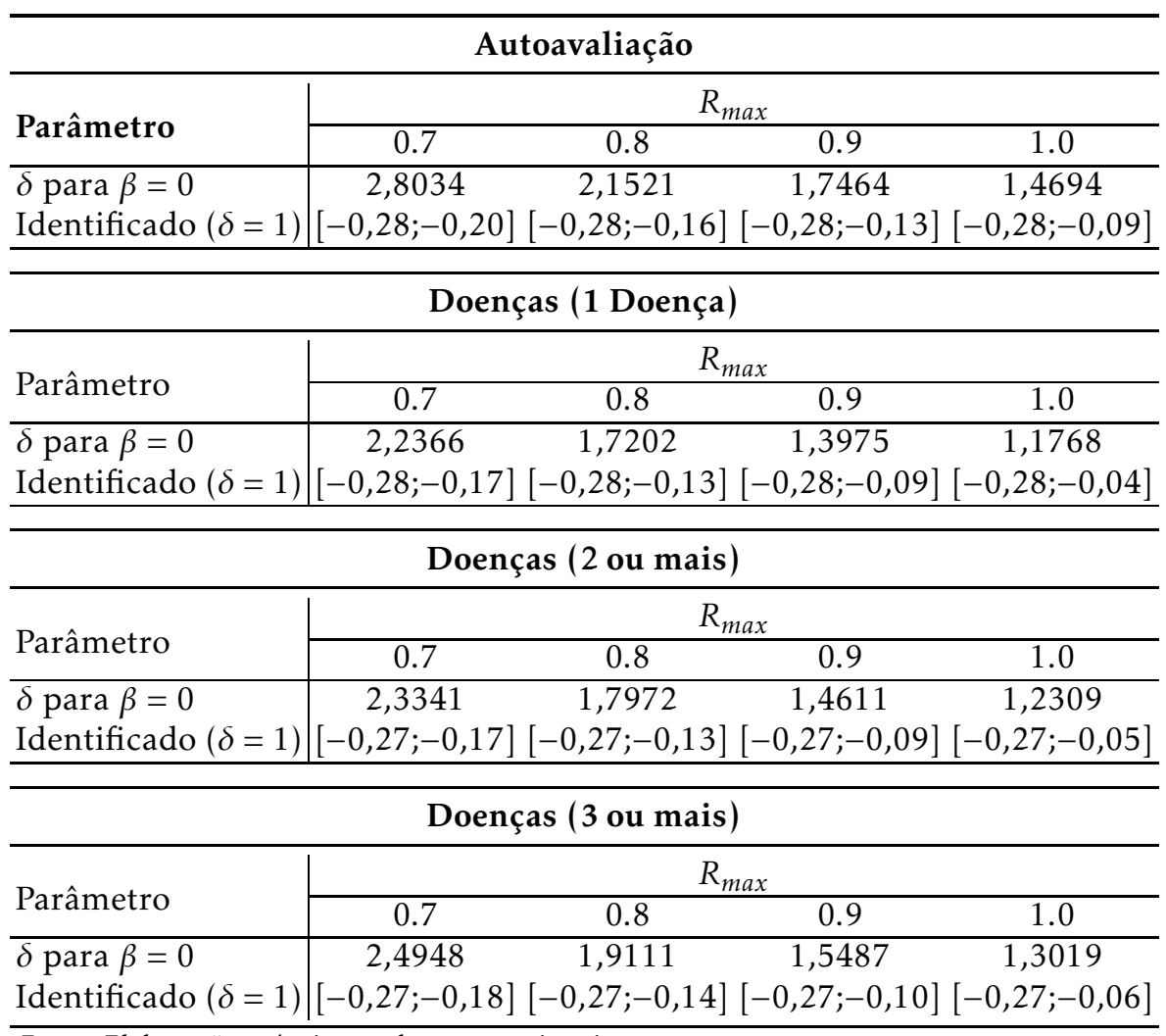

Fonte: Elaboração própria com base nas estimativas.

trou um coeficiente de proporcionalidade, $\delta$, de 1,37 e o conjunto identificado de $[-0,124 ;-0,033]$ que exclui o zero. Tal efeito mostrou-se significativo, inclusive quando comparado a outros métodos. Diante disso, tem-se uma confiabilidade nos resultados apresentados na presente pesquisa e corroborados nesta seção, sendo inclusive maiores que os valores encontrados por Oster (2015).

\section{Considerações Finais}

As condições de saúde, juntamente com a educação, são fatores primordiais para a acumulação de capital humano e consequentemente aumento da produtividade e aferição de renda. Ciente disso, foi estimado o impacto da má condição de saúde nos rendimentos do trabalho com dados da PNAD de 2008 que contém um suplemento de saúde, a partir de quatro métodos empíricos: efeito de tratamento médio e efeito de tratamento quantílico, ambos com correção semiparamétrica para o viés de seleção amostral; estimação a partir de instrumentos internos gerados pela heterocedasticidade dos erros do primeiro estágio seguindo Lewbel (2012), e a estimação dos bounds para o efeito de tratamento de Oster (2015), juntamente com o coeficiente de proporcionalidade que verifica o viés causado pelas variáveis não observáveis.

Foram utilizadas quatro variáveis indicadoras do status de saúde como tratamento como forma de corroborar as estimativas: a condição de saúde au- 
toavaliada, o diagnóstico de pelo menos uma doença por um profissional de saúde, o diagnóstico de duas ou mais doenças e o diagnóstico de três ou mais doenças. Os resultados mostraram um impacto negativo de uma má condição de saúde sobre os salários para todas as estimações, com uma possível superestimação do efeito médio quando comparado ao método de instrumentos internos de Lewbel (2012).

Quando a análise é estendida ao longo da distribuição de renda, é verificado que os indivíduos localizados nos quantis inferiores sofrem uma maior redução salarial devido a uma condição de saúde insatisfatória, o que pode agravar mais ainda a precariedade da saúde desses indivíduos, criando um "círculo vicioso" em tal relação, dado que os trabalhos que remuneram menos são em geral os que demandam maior esforço físico e, consequentemente, os mais afetados e de difícil adaptação por problemas de saúde, dada a impossibilidade de empreender o mesmo nível de esforço caso estivesse saudável.

As principais limitações do estudo se referem à análise em apenas um ponto no tempo, que impossibilita saber o estoque de saúde e informações sobre a entrada no mercado de trabalho dos indivíduos, e questões de causalidade reversa entre saúde e variáveis do mercado de trabalho. É possível que os indivíduos mais afetados por um nível de saúde insatisfatório já sofram reflexos por terem menos cuidados com a saúde por conseguirem apenas empregos de baixa qualificação e remuneração. No entanto, a estratégia empírica adotada, sobretudo o método de Lewbel a partir de instrumentos gerados internamente, dão uma maior confiabilidade aos resultados encontrados, visto que na população adulta a literatura têm mostrado que o canal de causalidade da saúde para a renda do trabalho é mais forte.

Por fim, o coeficiente de proporcionalidade, segundo Oster (2015), corrobora com os resultados anteriores e mostra que o viés causado pela omissão de variáveis ou por variáveis não observáveis não é capaz de tornar as estimativas insignificantes, servindo de robustez às estimações anteriores. Em suma, a má condição de saúde causa perdas salariais a todos os trabalhadores, sobretudo àqueles com menores rendimentos. Tais evidências reforçam a necessidade de mais oportunidades na área de saúde para esses trabalhadores, inclusive com prevenção e consultas/exames médicos de rotinas a fim de antecipar e tratar possíveis problemas e evitar uma baixa produtividade no mercado de trabalho.

\section{Referências Bibliográficas}

Abadie, A. \& Imbens, G. W. (2016), 'Matching on the estimated propensity score', Econometrica 84(2), 781-807.

Altonji, J. G., Elder, T. E. \& Taber, C. R. (2005), 'Selection on observed and unobserved variables: assessing the effectiveness of Catholic schools', Journal of Political Economy 113(1), 151-184.

Alves, L. \& Andrade, M. V. (2003), 'Impactos da saúde nos rendimentos individuais no Brasil', Revista de Economia Aplicada 7(2), 359-388.

Alves, L. F., Andrade, M. V. et al. (2002), 'Impactos do estado de saúde sobre os rendimentos individuais no Brasil e em Minas Gerais', X Seminário sobre a Economia Mineira, Anais p. 30. 
Amorim, G. \& Sampaio, B. (2015), Communication network and civil unrest: an investigation of the ocuppy movement in the United States, in '37th Meeting of the Brazilian Econometric Society, Brazil'.

Angrist, J. D. \& Pischke, J.-S. (2009), Mostly Harmless Econometrics: An Empiricist's Companion, Princeton University Press.

Austin, P. C. (2014), 'The use of propensity score methods with survival or time-to-event outcomes: reporting measures of effect similar to those used in randomized experiments', Statistics in Medicine 33(7), 1242-1258.

Barnay, T. (2016), 'Health, work and working conditions: a review of the European economic literature', The European Journal of Health Economics 17(6), 693-709.

Bartel, A. \& Taubman, P. (1979), 'Health and labor market success: the role of various diseases', The Review of Economics and Statistics pp. 1-8.

Baum, C. \& Schaffer, M. (2012), 'IVREG2H: Stata module to perform instrumental variables estimation using heteroskedasticity-based instruments'.

Böckerman, P., Hyytinen, A. \& Kaprio, J. (2015), 'Smoking and long-term labour market outcomes', Tobacco Control 24(4), 348-353.

Buchinsky, M. (1998), 'The dynamics of changes in the female wage distribution in the USA: a quantile regression approach', Journal of Applied Econometrics 13(1), 1-30.

Cacciamali, M. C. \& Hirata, G. I. (2005), 'A influência da raça e do gênero nas oportunidades de obtenção de renda - uma análise da discriminação em mercados de trabalho distintos: Bahia e São Paulo', Estudos Econômicos (São Paulo) 35(4), 767-795.

Cai, L. (2010), 'The relationship between health and labour force participation: evidence from a panel data simultaneous equation model', Labour Economics 17(1), 77-90.

Case, A. \& Deaton, A. S. (2005), Broken down by work and sex: how our health declines, in 'Analyses in the Economics of Aging', University of Chicago Press, pp. 185-212.

Coelho, D., Veszteg, R. \& Soares, F. V. (2010), Regressão quantílica com correção para a seletividade amostral: estimativa dos retornos educacionais e diferenciais raciais na distribuição de salários das mulheres no Brasil, Technical report, Texto para Discussão, Instituto de Pesquisa Econômica Aplicada (IPEA).

De Luca, G. \& Perotti, V. (2011), 'Estimation of ordered response models with sample selection', Stata Journal 11(2), 213-239.

De Luca, G. et al. (2008), 'SNP and SML estimation of univariate and bivariate binary-choice models', Stata Journal 8(2), 190.

Disney, R., Emmerson, C. \& Wakefield, M. (2006), 'Ill health and retirement in Britain: a panel data-based analysis', Journal of Health Economics 25(4), 621-649. 
Emran, M. S. \& Hou, Z. (2013), 'Access to markets and rural poverty: evidence from household consumption in China', Review of Economics and Statistics 95(2), 682-697.

Figueirêdo, L., Noronha, K. V. \& Andrade, M. V. (2003), 'Os impactos da saúde sobre o crescimento econômico na década de 90: uma análise para os estados brasileiros', Texto para Discussão (219).

Gallant, A. \& Nychka, D. W. (1987), 'Semi-nonparametric maximum likelihood estimation', Econometrica 55(2), 363-90.

García-Gómez, P., Jones, A. M. \& Rice, N. (2010), 'Health effects on labour market exits and entries', Labour Economics 17(1), 62-76.

García-Gómez, P., Van Kippersluis, H., O’Donnell, O. \& Van Doorslaer, E. (2013), 'Long-term and spillover effects of health shocks on employment and income', Journal of Human Resources 48(4), 873-909.

Godoy, M. R., Neto, G. B., Ribeiro, E. P. et al. (2006), 'Estimando as perdas de rendimento devido à doença renal crônica no Brasil', Programa de PósGraduação em Economia da Universidade Federal do Rio Grande do Sul .

Gomes, S. M. F. P. O., Brito, D. J. M. \& Rocha, R. M. (2014), Impactos da saúde sobre os rendimentos individuais no Brasil, in 'Anais do XL Encontro Nacional de Economia [Proceedings of the 40th Brazilian Economics Meeting]', number 242, ANPEC-Associação Nacional dos Centros de Pós-graduação em Economia [Brazilian Association of Graduate Programs in Economics].

Grossman, M. (1972), 'On the concept of health capital and the demand for health', Journal of Political Economy 80(2), 223-255.

Grossman, M. \& Benham, L. (1974), Health, hours and wages, in 'The Economics of Health and Medical Care', Springer, pp. 205-233.

Harris, A. (2009), 'Diabetes, cardiovascular disease and labour force participation in Australia: an endogenous multivariate probit analysis of clinical prevalence data', Economic Record 85(271), 472-484.

Heckman, J. (1979), 'Sample selection bias as a specification error', Econometrica $47(1)(161), 153$.

Heckman, J. J. \& Robb Jr, R. (1985), 'Alternative methods for evaluating the impact of interventions: an overview', Journal of Econometrics 30(1-2), 239267.

Ho, D. E., Imai, K., King, G. \& Stuart, E. A. (2007), 'Matching as nonparametric preprocessing for reducing model dependence in parametric causal inference', Political Analysis 15(3), 199-236.

Huber, M., Lechner, M. \& Wunsch, C. (2013), 'The performance of estimators based on the propensity score', Journal of Econometrics 175(1), 1-21.

Imbens, G. W. \& Wooldridge, J. M. (2009), 'Recent developments in the econometrics of program evaluation', Journal of Economic Literature 47(1), 5-86. 
Kassouf, A. (1999), 'Rendimentos perdidos por trabalhadores em condições inadequadas de saúde', Economia Aplicada, São Paulo 3(2), 239-262.

Kassouf, A. L. (1997), 'Saúde e mercado de trabalho'.

King, G. \& Nielsen, R. (2016), 'Why propensity scores should not be used for matching', Copy at http://j. mp/1sexgVw Download Citation BibTex Tagged XML Download Paper 378.

Koenker, R. (n.d.), 'Quantile regression, 2005'.

Koenker, R. \& Bassett Jr, G. (1978), 'Regression quantiles', Econometrica: Journal of the Econometric Society pp. 33-50.

Levine, P. B., Gustafson, T. A. \& Velenchik, A. D. (1997), 'More bad news for smokers? The effects of cigarette smoking on wages', ILR Review 50(3), 493509.

Lewbel, A. (2012), 'Using heteroscedasticity to identify and estimate mismeasured and endogenous regressor models', Journal of Business E Economic Statistics 30(1), 67-80.

Luft, H. S. (1975), 'The impact of poor health on earnings', The Review of Economics and Statistics pp. 43-57.

Lundborg, P., Nilsson, M. \& Vikström, J. (2015), 'Heterogeneity in the impact of health shocks on labour outcomes: evidence from swedish workers', Oxford Economic Papers 67(3), 715-739.

Mincer, J. (1974), 'Schooling, experience, and earnings. Human behavior \& social institutions n. 2'.

Morris, S. (2007), 'The impact of obesity on employment', Labour Economics 14(3), 413-433.

Noronha, K. V. M. S., Andrade, M. V. et al. (2004), A importância da saúde como um dos determinantes da distribuição de rendimentos e pobreza no Brasil, in 'Anais do XXXII Encontro Nacional de Economia [Proceedings of the 32th Brazilian Economics Meeting]', number 136, ANPEC-Associação Nacional dos Centros de Pós-graduação em Economia [Brazilian Association of Graduate Programs in Economics].

Oliveira, R., Moura, K., Viana, J., Tigre, R. \& Sampaio, B. (2015), 'Commute duration and health: empirical evidence from Brazil', Transportation Research Part A: Policy and Practice 80, 62-75.

Oliveira, V. R. (2014), 'Viés de seleção, migração e saúde', Revista Teoria e Evidência Econômica 20(43).

Oster, E. (2013), Unobservable selection and coefficient stability: theory and validation, Technical report, National Bureau of Economic Research.

Oster, E. (2015), 'Unobservable selection and coefficient stability: theory and evidence', Manuscript, Brown University. 
Pérez, E. R., Wajnman, S. \& Oliveira, A. (2006), 'Análise dos determinantes da participação no mercado de trabalho dos idosos em São Paulo', Revista Brasileira de Estudos de População 23(2), 269-286.

PNAD, I. (2008), 'Pesquisa nacional por amostra de domicílios, 2007'.

Rosenbaum, P. R. \& Rubin, D. B. (1983), 'The central role of the propensity score in observational studies for causal effects', Biometrika 70(1), 41-55.

Rosenbaum, P. R. \& Rubin, D. B. (1985), 'Constructing a control group using multivariate matched sampling methods that incorporate the propensity score', The American Statistician 39(1), 33-38.

Rubin, D. B. (1974), 'Estimating causal effects of treatments in randomized and nonrandomized studies', Journal of Educational Psychology 66(5), 688.

Saad, P. M. (1999), Transferência de apoio entre gerações no Brasil: um estudo para São Paulo e Fortaleza, in 'Muito Além dos 60: Os Novos Idosos Brasileiros', pp. 251-80.

Schultz, T. P. \& Tansel, A. (1997), 'Wage and labor supply effects of illness in cote d'ivoire and ghana: instrumental variable estimates for days disabled', Journal of Development Economics 53(2), 251-286.

Schultz, T. W. (1961), 'Investment in human capital', The American Economic Review 51(1), 1-17.

Silva, F. M. et al. (2011), 'Um estudo econômico da tuberculose no Brasil'.

Smith, J. P. (1999), 'Healthy bodies and thick wallets: the dual relation between health and economic status', Journal of Economic Perspectives 13(2), 145-166.

Sousa, E. A., dos Santos, A. M. A. \& Andrade Jacinto, P. (2010), 'Efeitos da educação sobre a saúde do indivíduo: uma análise para a região Nordeste do Brasil', Revista Econômica do Nordeste 44(4), 911-930.

Strauss, J. \& Thomas, D. (1998), 'Health, nutrition, and economic development', Journal of Economic Literature 36(2), 766-817.

Tigre, R., Sampaio, B. \& Menezes, T. (2017), 'The impact of commuting time on youth's school performance', Journal of Regional Science 57(1), 28-47.

Van Kippersluis, H., Van Ourti, T., O’Donnell, O. \& Van Doorslaer, E. (2009), 'Health and income across the life cycle and generations in Europe', Journal of Health Economics 28(4), 818-830.

Wilson, T. D. \& Brekke, N. (1994), 'Mental contamination and mental correction: unwanted influences on judgments and evaluations', Psychological Bulletin 116(1), 117. 
